\title{
6-phosphofructo-2-kinase/fructose-2,6-biphosphatase 4 is essential for p53-null cancer cells
}

Susana Ros ${ }^{1,8}$, Jessica Flöter ${ }^{2}$, Irem Kaymak ${ }^{2}$, Clive Da Costa ${ }^{3}$, Amina Houddane $^{4}$ Sébastien Dubuis ${ }^{5}$, Beatrice Griffiths ${ }^{1}$, Richard Mitter ${ }^{6}$, Susanne Walz $^{9}$, Sophia Blake ${ }^{3}$, Axel Behrens ${ }^{3,7}$, Kevin M. Brindle ${ }^{8}$, Nicola Zamboni ${ }^{5}$, Mark H. Rider ${ }^{4}$ and Almut Schulze ${ }^{1,2,9, \#}$

${ }^{1}$ Gene Expression Analysis Laboratory, Cancer Research UK London Research Institute, 44 Lincoln's Inn Fields, London WC2A 3LY, UK

${ }^{2}$ Theodor-Boveri-Institute, Biocenter, Am Hubland, 97074 Würzburg, Germany

${ }^{3}$ Mammalian Genetics Laboratory, Cancer Research UK London Research Institute, 44 Lincoln's Inn Fields, London WC2A 3LY, UK

${ }^{4}$ Université catholique de Louvain and de Duve Institute, Avenue Hippocrate 75, B-1200 Bruxelles, Belgium

${ }^{5}$ Institute of Molecular Systems Biology, ETH Zurich, Wolfgang-Pauli Str. 16 8093 Zurich, Switzerland

${ }^{6}$ Bioinformatics and Biostatistics Service, Cancer Research UK London Research Institute, 44 Lincoln's Inn Fields, London WC2A 3LY, UK

${ }^{7}$ School of Medicine, King's College London, Guy's Campus, London, SE1 1UL, UK

${ }^{8}$ Cancer Research UK Cambridge Institute, University of Cambridge, Cambridge, UK

${ }^{9}$ Comprehensive Cancer Center Mainfranken, Josef-Schneider-Str.6, 97080 Würzburg, Germany

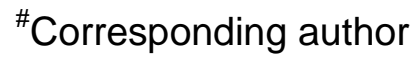

Running Title: p53 regulates PFKFB4 in cancer 


\section{ABSTRACT}

The bifunctional enzyme 6-phosphofructo-2-kinase/fructose-2,6biphosphatase-4 (PFKFB4) controls metabolic flux through allosteric regulation of glycolysis. Here we show that p53 regulates the expression of PFKFB4 and that p53-deficient cancer cells are highly dependent on the function of this enzyme. We found that p53 down-regulates PFKFB4 expression by binding to its promoter and mediating transcriptional repression via histone deacetylases. Depletion of PFKFB4 from p53 deficient cancer cells increased levels of the allosteric regulator fructose 2,6-bisphophate, leading to increased glycolytic activity but decreased routing of metabolites through the oxidative arm of the pentose phosphate pathway. PFKFB4 was also required to support the synthesis and regeneration of nicotinamide adenine dinucleotide phosphate (NADPH) in p53 deficient cancer cells. Moreover, depletion of PFKFB4 attenuated cellular biosynthetic activity and resulted in the accumulation of reactive oxygen species and cell death in the absence of p53. Finally, silencing of PFKFB4 induced apoptosis in p53 deficient cancer cells in vivo and interfered with tumour growth. These results demonstrate that PFKFB4 is essential to support anabolic metabolism in p53deficient cancer cells and suggest that inhibition of PFKFB4 could be an effective strategy for cancer treatment.

Keywords: cancer metabolism; p53; PFKFB4; fructose 2,6-bisphosphate; NADPH; pentose phosphate pathway; tumour growth 


\section{INTRODUCTION}

Cancer cells need to generate large amounts of metabolic precursors to support macromolecule biosynthesis during cell growth and proliferation. Cancer cell metabolism is characterised by enhanced nutrient uptake and increased activity of many biosynthetic processes, including protein and lipid biosynthesis. Nicotinamide adenine dinucleotide phosphate (NADPH) is a cofactor for anabolic reactions and required for the regeneration of reduced glutathione (GSH) for the detoxification of reactive oxygen species (ROS). The enhanced demand for biosynthetic processes and control of oxidative stress makes NADPH synthesis and regeneration an essential process in cancer $^{26}$.

The tumour suppressor p53 counteracts many of the metabolic alterations observed in cancer cells ${ }^{21}$. Indeed, modulation of metabolic activity was shown to be a major component of the tumour suppressor activity of p53 ${ }^{25}$. Loss of p53 function leads to enhanced glucose uptake ${ }^{19}$ but decreased mitochondrial oxidative metabolism ${ }^{29}$. Activation of p53 by DNA damage induces the expression of TIGAR, a fructose-2,6-bisphosphatase domain containing protein, which reduces glycolytic activity and increases the flux of metabolites to the oxidative pentose phosphate pathway (PPP) ${ }^{2}$ to facilitate nucleotide synthesis for DNA repair. Seemingly contradictory to this, loss of p53 in cancer cells enhances oxidative PPP activity by increasing the activity of glucose-6-phosphate dehydrogenase (G6PD) and 6-phosphogluconate dehydrogenase (6PGD), the two NADPH-generating enzymes of this pathway 12, 17. p53 also regulates the expression of malic enzymes 1 and 2 (ME1, ME2), two additional NADPH producing metabolic enzymes ${ }^{18}$. It can therefore be concluded that deletion of p53 overall increases the synthesis and regeneration of NADPH to support biosynthetic processes in cancer cells. We have previously shown that 6-phosphofructo-2-kinase/fructose-2,6biphosphatase-4 (PFKFB4) supports the survival of prostate cancer cells in vitro and in vivo by reducing the generation of the allosteric regulator fructose 2,6-bisphosphate (Fru-2,6-BP), thereby inhibiting glycolytic flux and promoting the production of NADPH for lipid synthesis and regeneration of GSH ${ }^{35}$. Silencing of PFKFB4 blocked acetate-dependent lipid synthesis and resulted 
in the accumulation of ROS in cancer cells ${ }^{35}$. However, it was not known whether loss of p53 function alters the dependency of cancer cells on allosteric regulation of glycolysis by PFKFB4, thereby postulating a potential biomarker for therapeutic targeting strategies.

We now demonstrate that p53 negatively regulates PFKFB4 expression by directly binding to the promoter of the gene. Conversely, loss of p53 function either by deletion of mutation increases the expression of PFKFB4 in cancer cells. Cancer cells that have lost p53 function are also highly sensitive to PFKFB4 depletion. Allosteric regulation of glycolysis by PFKFB4 is required for the routing of metabolites to the oxidative PPP to maintain NADPH synthesis and regeneration. Depletion of PFKFB4 in p53 deficient cancer cells increases oxidative stress and results in the induction of apoptosis. This dependence was also observed in xenograft tumours, where silencing of PFKFB4 caused more efficient blockade of tumour growth of p53 negative cancer cells. Our results reveal that inhibition of PFKFB4 interacts in a synthetic lethal manner with loss of function of p53 in cancer cells and provide a rationale for targeting allosteric regulation of glycolysis for pharmaceutical intervention in cancers that are defective in TP53.

\section{RESULTS}

\section{p53 negatively regulates PFKFB4 expression}

To investigate whether PFKFB4 is regulated by $\mathrm{p53}$, we analysed expression of the four human PFKFB isoforms in isogenic $\mathrm{p} 53^{+/+}$and $\mathrm{p} 53^{-{ }^{-}}$HCT116 colon cancer cells. mRNA levels of PFKFB4, but not any of the other PFKFB isoforms, were markedly increased in $\mathrm{p} 53^{-/-}$cells compared to their wild type counterparts (Fig. 1a). This induction was similar to the increased expression of malic enzyme 1 (ME1) and malic enzyme 2 (ME2), which were previously identified as negative targets of p53 ${ }^{18}$, while expression of TIGAR was not altered between $\mathrm{p} 53^{+/+}$and $\mathrm{p} 53^{-/-}$cells (Fig. 1b). In agreement with altered mRNA levels, we also detected increased expression of PFKFB4 protein in HCT116 p53 ${ }^{-/}$cells (Fig. 1c), while the other PFKFB isoforms showed no differential expression (Fig. S1a). Moreover, CRE-mediated deletion of p53 in primary embryonic fibroblasts (MEF) derived from tpr53 ${ }^{\text {flffl}}$ mice also resulted 
in increased Pfkfb4 expression (Fig. 1d). Silencing of TP53 in HCT116 p53 cells, using a lentiviral vector for the doxycycline-inducible expression of short hairpin RNA (shRNA) targeting p53, efficiently reduced the expression of $p 53$ and $p 21$ but increased expression of PFKFB4 and ME2 (Fig. 1e). Conversely, expression of $p 53$ in the p53 deficient ovarian cancer cell line SKOV3 caused a marked decrease in the expression of PFKFB4 mRNA and protein (Fig. If and $\mathrm{g}$ ).

The normally short-lived p53 protein is stabilized in response to DNA-damage

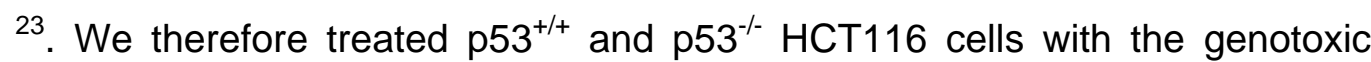
agent etoposide and monitored changes in expression of p53 target genes. Etoposide caused a dose-dependent induction in p21 expression only in

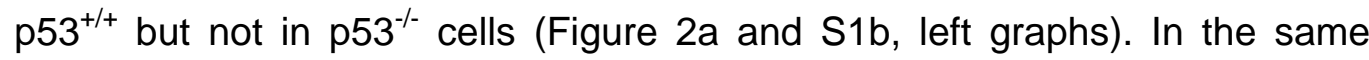
cells, expression of PFKFB4 was reduced in response to etoposide treatment only in the presence of p53+/+ (Fig. $2 a$ and S1b, middle graphs). This was similar to the regulation of ME2 (Fig. 2a and S1b, right graphs). Similar results were also obtained in a second p53 wild type colon cancer cell line, LOVO (Fig. 2b), and a p53 wild type breast cancer cell line, MCF7 (Fig. 2c).

Analysis of the human PFKFB4 promoter revealed several putative p53response elements. Chromatin immunoprecipitation (ChIP) experiments showed specific binding of p53 to two of these sites (Fig. 2d), confirming that p53 regulates PFKFB4 expression by binding to its promoter. Negative regulation of transcription by $p 53$ frequently involves the recruitment of histone deacetylases (HDACs) to the promoter regions of target genes ${ }^{13,31}$. We therefore investigated whether inhibition of HDACs by trichostatin A (TSA) prevents the negative effect of p53 on PFKFB4 expression. TSA treatment efficiently blocked PFKFB4 downregulation in the presence of etoposide in

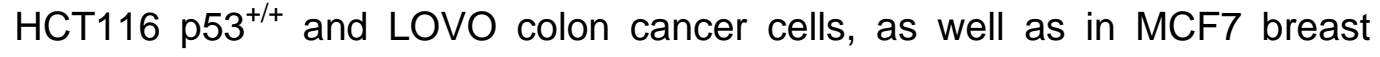
cancer cells (Fig. 2e).

Taken together, these results demonstrate that p53 negatively regulates expression of PFKFB4, both at basal levels and when p53 is activated in response to DNA damage. To confirm the link between PFKFB4 expression 
and p53 also in human cancer, we interrogated public datasets from the Gene Expression Omnibus (GEO). We retrieved expression data of cancer samples with differential p53 status from a study investigating somatic mutations in lung adenocarcinoma ${ }^{8}$. Comparative analysis revealed that PFKFB4

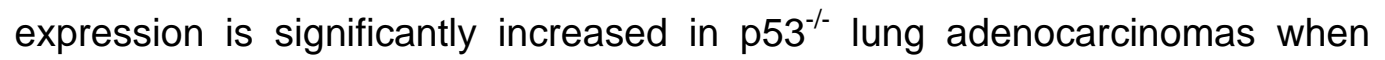
compared to ${\mathrm{p} 53^{+/+}}^{+}$tissues of the same cancer type (Fig. S1c).

\section{PFKFB4 is essential for the viability of p53 null cancer cells}

PFKFB proteins are homodimeric bifunctional enzymes that control the rate of glycolysis by modulating levels of Fru-2,6-BP, an allosteric activator of phosphofructokinase 1 (PFK1) and inhibitor of fructose-1,6-bisphosphatase (FBPase-1) ${ }^{32}$. Allosteric regulation by PFKFB4 regulates the distribution of metabolites between glycolysis and the oxidative PPP and is required for the generation of NADPH for anabolic reactions and anti-oxidant synthesis in cancer cells ${ }^{35,39}$.

To investigate whether the increased expression of PFKFB4 is linked to the altered metabolic demand of ${\mathrm{p} 53^{-/}}^{-}$cancer cells, we performed silencing experiments using inducible expression of shRNAs targeting PFKFB4. Depletion of PFKFB4 using two independent shRNA hairpins only

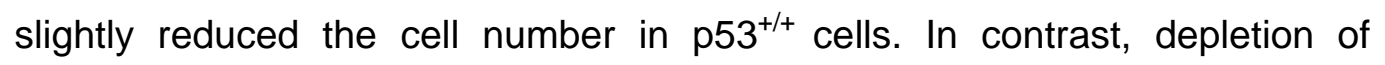
PFKFB4 caused a substantial reduction of viability in ${\mathrm{p} 53^{\prime}}^{\prime}$ cells (Fig. 3a).

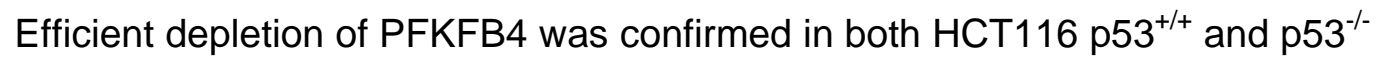
cell lines, with sequence \#68 achieving an 80\% reduction in mRNA while expression of sequence \#64 resulted in a 50\% reduction (Fig. 3b). We also controlled that depletion of PFKFB4 did not cause any changes in the expression of PFKFB1, PFKFB2 or PFKFB3 (Fig. S2a). Furthermore, to establish specificity of silencing, we generated shRNA-insensitive expression constructs by introducing silent mutations within the PFKFB4 coding

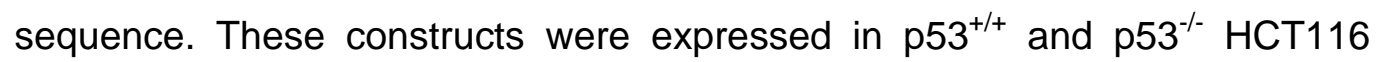
colon cancer cells, which were subsequently infected with the lentiviral shRNA expression constructs targeting endogenous PFKFB4. We confirmed that the expression of exogenous PFKFB4 mRNA was not changed upon doxycycline 
treatment (Fig. S2b and c). Importantly, reduced viability of HCT116 p53-cells in response to shRNA induction was completely rescued by reexpression of PFKFB4 (Fig. 3c).

We next investigated the wider applicability of these findings using a panel of colon cancer cell lines. These included three additional p53 wild type colon cancer cell lines (RKO, LOVO and LS174T) and two colon cancer cell lines (SW680 and SW620) expressing a mutant form of p53 carrying a mutation within the DNA binding domain $(\mathrm{R} 273 \mathrm{H})$. This mutant exerts a dominant negative effect by blocking DNA binding of the wild type protein ${ }^{43}$. Wild type cell lines expressed significantly lower levels of PFKFB4, with RKO cells expressing nearly undetectable levels (Fig. $3 d$ and e). We next transduced two p53 wild type and p53 mutant cell lines with lentiviral shRNA constructs targeting PFKFB4. Depletion of PFKFB4 reduced the viability of both p53 mutant cell lines, while not affecting viability of cells expressing wild type p53 (Fig. 3e and f). Conclusively, these results confirm that PFKFB4 is selectively required for the viability of p53 deficient cancer cells.

\section{PFKFB4 maintains balance of metabolite flux between glycolysis and pentose phosphate pathway in p53 deficient cancer cells}

We next asked which catalytic activity of PFKFB4 mediates the survival of $\mathrm{p5}^{-/-}$cells. We first established the efficiency and timing of PFKFB4 protein depletion in response to shRNA expression. While mRNA levels were already efficiently reduced after 2 days of doxycycline treatment in cells expressing sequence \#68, substantial amounts of protein were still detectable after 3 days of shRNA induction (Fig. S3a and b). Moreover, p53 deficient cells showed a larger increase in PFKFB4 protein expression at the later time point (Fig. 4a and S3d). We next determined the levels of Fru-2,6-BP, the substrate of the fructose-2,6-bisphosphatase activity of PFKFB4 at 3 and 6 days after PFKFB4 depletion in both cell types. At the earlier time point, depletion of PFKFB4 caused an increase in Fru-2,6-BP in both $\mathrm{p} 53^{+/+}$and $\mathrm{p} 53^{\prime}$ cells (Fig. $\mathrm{S} 3 \mathrm{c}$ ). However, at the later time point, where $\mathrm{p5} 3^{\prime}$ cells show increased expression of the protein, no changes in metabolite levels were observed in 
p53 ${ }^{+/+}$cells. In contrast, depletion of PFKFB4 for 6 days caused a significant increase in Fru-2,6-BP levels in p53 deficient cells (Fig. 4b). Induction of the second, less effective, shRNA sequence only caused partial ablation of PFKFB4 protein after 6 days, which also increased the levels of Fru-2,6-BP only in p53 ' cells, although the data failed to reach statistical significance (Fig. S3e).

To further investigate the consequences of PFKFB4 depletion on cellular metabolism, we examined changes in glucose uptake using 2-[N-(7-nitrobenz2-oxa-1,3-diazol-4-yl) amino]-2-deoxy-D-glucose (2-NBDG), a fluorescent derivative of 2-deoxyglucose. p53 inhibits glucose uptake by downregulating the expression of glucose transporters GLUT1 and GLUT4 ${ }^{38}$. Moreover, activation of p53 suppresses NF- B activation and reduces glucose uptake and glycolysis by blocking the expression of GLUT3 ${ }^{19}$. However, we observed no major differences in 2-NBDG uptake between $\mathrm{p} 53^{+/+}$and $\mathrm{p} 53^{-/-}$ cells. Depletion of PFKFB4 caused a moderate increase in 2-NBDG uptake in both cell lines, which was significant only in p53/- cells (Fig. S3f).

To provide further detail of the metabolic response to PFKFB4 depletion, we also performed mass isotopomer distribution (MID) analysis after exposing cells to $\left[1,2-{ }^{13} \mathrm{C}\right]$-glucose that allows us to estimate the relative contribution of the PPP to the metabolite pool in glycolysis. Specifically, molecules with one ${ }^{13} \mathrm{C}$ atom $(\mathrm{M}+1)$ indicate that they transited through the oxidative PPP, whereas molecules with two ${ }^{13} \mathrm{C}$ atoms $(\mathrm{M}+2)$ came directly from glycolysis (Fig. S3g). We analysed the mass isotopomer fractions of fructosebisphosphate (FBP) and phosphoenolpyruvate (PEP), and found that silencing of PFKFB4 in $\mathrm{p53}^{-{ }_{-}}$HCT116 cells leads to an increased fraction of $\mathrm{M}+1$ over the $\mathrm{M}+2$ fraction for both FBP and PEP (Fig. S3h), that means a relative increase in the fraction of pentose phosphates that is routed back to glycolysis through the non-oxidative PPP. The most plausible explanation for this result is that PFKFB4 depletion causes a difference in the outflow of pentose-phosphate for purine biosynthesis, which is the major biosynthetic

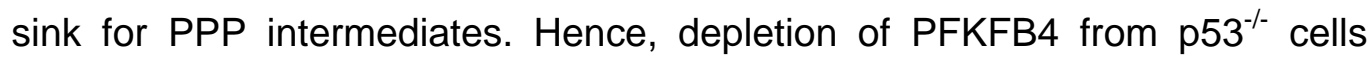


induces a reduction in the net flux through the oxidative PPP from glucose-6phosphate to the pentose-phosphate pool, but the latter is seemingly channelled back because of a reduced outflow to purine synthesis.

MID analysis is not able to provide any estimation of the rate at which metabolites pass through a metabolic pathway. We next investigated the effect of PFKFB4 depletion on glycolytic flux (ECAR) and oxygen consumption (ORC) using the Seahorse Bioanalyzer. Both cell lines showed similar ECAR/OCR ratios in the basal state and depletion of PFKFB4 had only

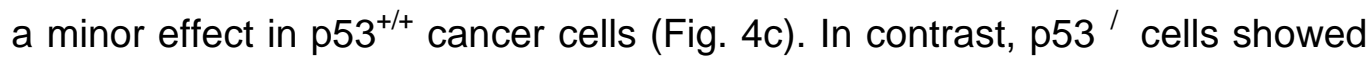
a strong increase in the ECAR/OCR ratio upon PFKFB4 depletion (Fig. 4c). This was caused by an increase in glycolysis and a decrease in respiration (Fig. 4d), confirming that PFKFB4 is required to limit glycolytic flux in these cells.

Increased glycolysis affects metabolite flux towards the oxidative and nonoxidative arm of the PPP. We therefore analysed the activity of the oxidative PPP by comparing the production of ${ }^{14} \mathrm{CO}_{2}$ from $1-{ }^{14} \mathrm{C}$-glucose relative to that from $6-{ }^{14} \mathrm{C}$-glucose. We observed a significant reduction of this ratio in HCT116 p53 $^{-1-}$ cells after silencing of PFKFB4 (Fig. 4e). In contrast, silencing

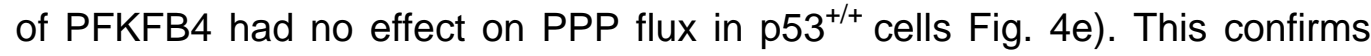
that PFKFB4 is required to maintain the activity of the oxidative arm of the PPP in p53 deficient cancer cells.

\section{PFKFB4 maintains NADPH production for biosynthetic activity and antioxidant production}

The metabolic outputs of the PPP include the synthesis of riboses for nucleotide biosynthesis and the regeneration of $\mathrm{NADPH}$. We therefore investigated whether PFKFB4 influences NADPH levels and oxidation status in cancer cells. Interestingly, we found that levels of NADPH as well as the total amount of this coenzyme (NADPH+NADP ${ }^{+}$) where reduced following PFKFB4 silencing only in $\mathrm{p5}^{- \text {- }}$ cells (Fig. $5 \mathrm{a}$, left graph). In contrast, the ratio between the reduced and oxidised forms of the coenzyme was not altered 
(Fig. 5a, right graph). Moderate depletion of PFKFB4 protein achieved by the second, less efficient sequence also reduced NADPH levels (Fig. S4a). This effect was only detectable at the later time point, when efficient depletion of PFKFB4 protein was achieved (Fig. S4a), while short-term silencing had no effect (Fig. S4b). We additionally determined expression of ME1 and ME2 after PFKFB4 knockdown and observed no compensation by these genes (Fig. S4c). In agreement with the results of the MID analysis, this result suggests that PFKFB4 is required to maintain NADPH regeneration through the oxidative arm of the PPP and also facilitates the synthesis of the coenzyme by providing riboses via the biosynthetic output of this pathway.

NADPH provides reducing power for biosynthetic reactions, including lipid biosynthesis, leading us to investigate whether inhibition of PFKFB4 affects the activity of this process in cancer cells. We first established that $\mathrm{p} 53^{-{ }^{-}}$cells display a higher rate of acetate-dependent lipid biosynthesis compared to p53 ${ }^{+/+}$cells (Fig. 5b). Moreover, p53 deficient cells also showed increased activity of a reporter containing a fragment of the human HMG-CoA synthase promoter carrying an intact sterol regulatory element (SRE) (Fig. 5c). This activity depends on the sterol regulatory element binding proteins (SREBP), a family of transcription factors controlling genes involved in fatty acid and cholesterol biosynthesis ${ }^{1}$. This is in agreement with previous reports showing that SREBP is activated by loss or mutation of p53 ${ }^{10,44}$. It is therefore possible that p53 deficient cancer cells have a higher demand for NADPH than their p53 proficient counterparts, which is aided by the induction of PFKFB4. In agreement with this hypothesis, silencing of PFKFB4 caused a stronger reduction in acetate-dependent lipid synthesis in ${\mathrm{p} 53^{-/-}}^{-}$cells

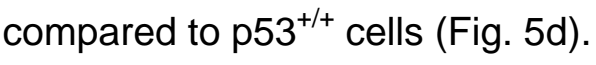

Increased NADPH demand also compromises the availability of this coenzyme for the production of antioxidants, leading to oxidative damage and ROS generation. We therefore investigated the effect of PFKFB4 depletion on cellular ROS levels in the two cell lines. While silencing of PFKFB4 had no effect on ROS in $\mathrm{p53}^{+/+}$cells, it caused a strong increase in ROS levels in p53 ' cells (Fig. 5e). Furthermore, treatment with the antioxidant $\mathrm{N}$ - 
acetylcysteine (NAC) blocked the induction of apoptosis in response to PFKFB4 depletion in $\mathrm{p53}^{-/-}$cells (Fig. 5f), indicating that ROS accumulation is the cause of the reduced viability observed in these cells.

Taken together, these results confirm the role of PFKFB4 in promoting the routing of metabolites from glycolysis into the PPP for the synthesis and regeneration of NADPH to support anabolic reactions and maintain cellular redox balance. This function is essential to fulfil the increased NADPH demand of p53 deficient cancer cells to drive biosynthetic processes including lipid synthesis (Fig. 5g).

\section{PFKFB4 silencing inhibits spheroid and tumour growth in the absence of p53}

Metabolic reprogramming in cancer supports cell growth under oxygen and nutrient limited conditions, such as those found in hypo-vascularised tumour regions ${ }^{7,30}$. The environmental conditions found in tumours can generate specific metabolic constraints and reveal sensitivities of cancer cells that could be exploited for therapy.

To investigate the contribution of PFKFB4 to the viability of p53 deficient cancer cells under nutrient limited conditions, we employed tumour spheroid culture conditions. These multi-layered 3-dimensional structures recreate nutrient and oxygen gradients found in live tumours ${ }^{5}$. Silencing of PFKFB4 induced a reduction in spheroid volume in both $\mathrm{p53}^{+/+}$and $\mathrm{p} 53^{-/-}$HCT116 colon cancer cells (Fig. S5a), indicating that the metabolically compromised conditions present in this culture system could sensitise $\mathrm{p} 53^{+/+}$cells towards PFKFB4 inhibition. However, depletion of PFKFB4 caused a further reduction in spheroid growth in $\mathrm{p} 53^{-/-}$cells, confirming the increased dependency of the cells on this enzyme in the absence of the tumour suppressor.

We next investigated the importance of PFKFB4 for tumour growth using a

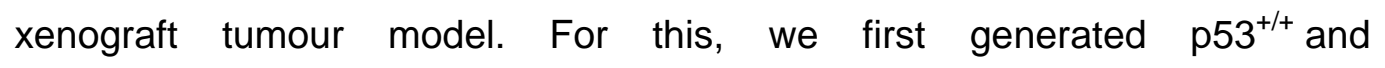
p53 ' HCT116 cells expressing luciferase and used these for expression of inducible shRNA-constructs targeting PFKFB4 or scrambled shRNA controls. 
Efficient PFKFB4 silencing and inhibition of cell proliferation was confirmed in these cells (Fig. S5b and c). Cells were then injected subcutaneously into nude mice and tumour growth was followed over time in control and doxycycline treated cohorts. As control, we established that PFKFB4 silencing had no effect on the size of tumours generated by HCT116 cells expressing a scrambled shRNA control (Fig. S5d and e).

Interestingly, we found that PFKFB4 mRNA levels were higher in $\mathrm{p} 53^{-/-}$ tumours, confirming that the differential expression of this gene is also maintained in vivo, and that doxycycline treatment efficiently depleted PFKFB4 mRNA from both types of tumours (Fig. S5f). Strong silencing of PFKFB4 by sequence \#68 slowed the growth of $\mathrm{p} 53^{+/+}$tumours, with a plateau being reached after 15 days of doxycycline treatment. Induction of the

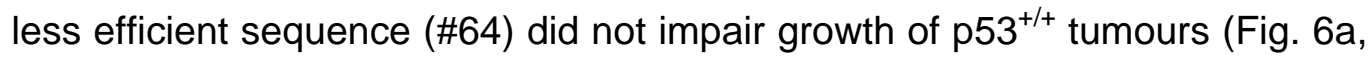
left graphs). In contrast, expression of either shRNA sequences efficiently blocked tumour growth in the p53 ' background. Indeed, strong silencing by sequence \#68 caused a reduction of the initial tumour mass and a 17-fold reduction of tumour size compared to untreated controls at the end of the experiment (Fig. 6a, right graphs). Consequently, the effects of PFKFB4 depletion on bioluminescence detection (Fig. 6b), tumour weight (Fig. S5d) and tumour size (Fig. S5e) were more pronounced in p53 ' background.

Histological analysis showed that tumour regression was accompanied by the appearance of apoptotic areas (Fig. 6c), while minor changes in proliferation were detected (Fig. 6d and S5g). We also analysed transcriptional changes associated with PFKFB4 inhibition in these tumours using whole genome

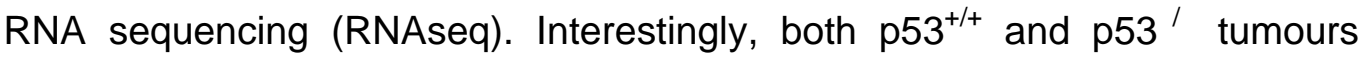
showed downregulation of genes associated with ribosome synthesis, assembly of the ternary translation complex and rapamycin sensitivity (Fig. 6e and S6a). However, only p53 ' tumours showed regulation of genes linked to apoptosis and oxidative stress (Fig S6a). Finally, analysis of public gene expression data revealed that high expression of the glycolytic enzyme PFKFB4 is a predictor of reduced survival in breast and non-small cell lung cancer patients (Fig. S6b and c). 
In conclusion, our study demonstrates that p53 represses PFKFB4 expression, and that allosteric regulation of glycolytic flux by PFKFB4 supports lipid synthesis and prevents oxidative stress in p53 deficient cancer cells to support cell proliferation and tumour growth.

\section{DISCUSSION}

Loss of p53 in cancer cells inactivates important DNA-damage and cell cycle checkpoints but has also multiple effects on cellular metabolism ${ }^{27}$. Indeed, the metabolic effects of p53 may be sufficient to explain its functions as a tumour suppressor ${ }^{25}$. In normal cells, p53 regulates metabolism on multiple levels ${ }^{21}$. For example, p53 reduces glucose uptake by lowering NF- B dependent expression of GLUT3 ${ }^{19}$. Induction of p53 in response to DNA damage inhibits glycolysis through the induction of TIGAR ${ }^{2}$. It also reduces the expression of several glycolytic enzymes via a microRNA-dependent mechanism ${ }^{20}$ and increases mitochondrial activity by inducing the expression of cytochrome c oxidase 2 (SCO2) ${ }^{29}$. At the same time, p53 also increases the ability of cells to respond to nutrient starvation, by facilitating cell cycle arrest, autophagy or the use of alternative energy source, such as lipids ${ }^{21}$. Cancer cells deficient in p53 can therefore be more sensitive towards perturbations within the metabolic network and these selective vulnerabilities can be exploited for cancer therapy ${ }^{28}$.

Enhanced macromolecule biosynthesis increases the cellular demand for $\mathrm{NADPH}$, the reducing coenzyme for anabolic reaction. Cancer cells therefore need to upregulate mechanisms that increase NADPH synthesis and regeneration to maintain rapid proliferation and prevent excess oxidative stress. We demonstrate that p53 negatively regulates expression of PFKFB4, while loss of p53 increases the expression of PFKFB4, an enzyme that controls cellular levels of the allosteric regulator Fru-2,6-BP. Through this regulation, p53 alters the routing of metabolites between glycolysis and the pentose phosphate pathway, which is critical for NADPH synthesis and regeneration. Depletion of PFKFB4 impairs the ability of cancer cells to 
generate sufficient amounts of reducing cofactors to fulfil the demand generated by increased lipid synthesis. As a result, inhibition of PFKFB4 selectively increases oxidative stress in p53 deficient cancer cells, leading to reduced viability in vitro and tumour regression in vivo.

Several mechanisms have been proposed for the negative regulation of gene expression by $\mathrm{p} 53$. These include competition for DNA binding or transactivation function of positive regulators of transcription ${ }^{34}$. We found that p53 directly binds to two p53-response elements within the PFKFB4 promoter. Moreover, we found that activation of p53 by DNA damaging agents reduced PFKFB4 expression, which was blocked by addition of TSA, suggesting that p53 represses transcription by recruiting HDACs. Loss of p53 relieves this inhibition and allows increased expression of PFKFB4.

Allosteric regulation of glycolytic activity by PFKFB proteins is an important principle for the control of metabolic flux in cancer cells ${ }^{36}$. PFKFB proteins have two independent catalytic centres and it is important to establish the exact role of PFKFB4 within the metabolic network of cancer cells. We have previously shown that PFKFB4 limits Fru-2,6-BP levels and induce the routing of metabolites into the PPP to support NADPH production and ROS detoxification in prostate cancer cells ${ }^{35}$. In contrast, a recent study observed that PFKFB4 promotes the synthesis of Fru-2,6-BP to increase glycolytic flux in cancer cells grown under hypoxic conditions or as xenograft tumours ${ }^{6}$. Here we show that depletion of PFKFB4 increases the levels of Fru-2,6-BP in p53 deficient colon cancer cells. Mass isotopomer distribution analysis confirmed that depletion of PFKFB4 increases the relative fraction of pentosephosphates that are routed back to glycolysis from the non-oxidative PPP. We also found that PFKFB4 depletion increases glycolytic rate and decreased activity of the oxidative arm of the PPP. Together, these metabolic changes results in reduced availability of PPP intermediates for biosynthetic processes, such as the production of $\mathrm{NADPH}$, an essential cofactor for biosynthetic reaction. Indeed, NADPH levels were dramatically reduced following PFKFB4 depletion. This resulted in the selective induction of oxidative stress and cell death in p53 deficient cancer cells. 
There are several factors that contribute to the exquisite sensitivity of p53 deficient cancer cells towards PFKFB4 depletion. One is the increased biosynthetic demand of p53 deficient cancer cells. p53 was shown to decrease the expression of lipogenic genes in adipocytes of obese mice ${ }^{44}$. Loss of p53 increases the activity of $\operatorname{mTORC} 1{ }^{9}$, which drives both protein and lipid biosynthesis ${ }^{22}$. As lipid biosynthesis requires large amounts of NADPH, regulatory mechanisms that support the production of this coenzyme must be upregulated when lipid biosynthesis is high. Another explanation could be that p53 deficient cancer cells fail to undergo cell cycle arrest under metabolic starvation conditions, such as the absence of exogenous serine ${ }^{28}$. It is therefore likely that p53 deficient cells are unable to adapt to the altered metabolite flux following PFKFB4 depletion by efficiently downregulating NADPH-consuming biosynthetic processes. This would cause further NADPH depletion, leading to oxidative stress and cell death. In line with this hypothesis, the reduction in tumour growth observed after strong PFKFB4 depletion in p53 proficient cancers was not associated with the induction of cell death. Remarkably, p53 proficient cancer cells were not affected in their ability to form xenograft tumours when PFKFB4 was partially depleted using a less efficient shRNA sequence. In contrast, p53 deficient cancer cells were highly dependent on PFKFB4, as both partial and efficient silencing blocked tumour growth. Moreover, depletion of PFKFB4 from p53 deficient tumours resulted in the induction of apoptosis and tumour regression.

Dynamic regulation of carbohydrate flux is essential to counteract oxidative stress in yeast ${ }^{33}$. In cancer, the control of NADPH synthesis and regeneration to maintain anabolic metabolism and antioxidant production is an essential function of drivers of oncogenic transformation. The results of this study demonstrate that allosteric regulation of the glycolytic flux by PFKFB4 is essential for the survival of $\mathrm{p} 53$ deficient cancer cells. This synthetic lethality suggests that targeting PFKFB4 could be an effective strategy for the treatment of tumours that have lost the function of this important tumour suppressor. 


\section{MATERIALS AND METHODS}

\section{Cell culture and reagents}

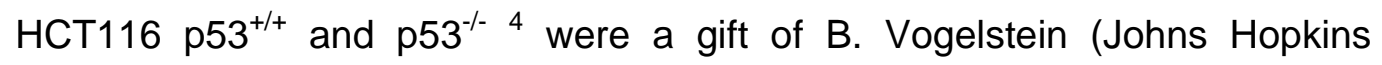
University, Baltimore). HEK 293T, MCF7, LOVO, LS174T, SW480 and SW620 cells were from LRI Cell Services. SKOV3, and also MCF7, LOVO were from CRUK-Cl Biorepository Services. All cell lines were authenticated by STR profiling and used at low passage. All cell lines were confirmed to be free from mycoplasma throughout the experiments by regular testing. Cells were cultured at $37^{\circ} \mathrm{C}$ in a humidified incubator at $5 \% \mathrm{CO}_{2}$ in Dulbecco's modified Eagle's medium (DMEM, Life Technologies) with 10\% foetal bovine serum (FBS, Sigma) and 2mM L-glutamine. Primary MEF were derived from embryonic day 12.5 (E12.5) embryos and were cultured in DMEM supplemented with $10 \%$ FBS at $37^{\circ} \mathrm{C}$ with $5 \% \mathrm{CO}_{2}$ and $3 \% \mathrm{O}_{2}$. For Cremediated deletion of $\mathrm{p} 53$ floxed alleles, MEF from $\mathrm{tp} 53^{\mathrm{fl} / \mathrm{fl}}$ mice were infected with Adeno-Cre-green fluorescent protein (GFP) or Adeno-GFP virus (Gene Transfer Vector Core, lowa University) for $48 \mathrm{hr}$. Virus was removed and the cells were allowed $48 \mathrm{hr}$ to recover before passaging.

Antibodies for PFKFB1 (SAB1408617) and PFKFB2 (SAB1406248) were from Sigma, for PFKFB3 (H00005209-A01) from Abnova and PFKFB4 (ab137785) from Abcam or from Abgent (Center, AP815c). Antibodies for p53 (DO-1, sc$126 \mathrm{x}$ ) were from Santa Cruz Biology. All other reagents were from Sigma. The HMGCS promoter constructs pGL3-SYNwt-luc and pGL3-SYNmutSRE1 were a gift from J. Swinnen (Catholic University, Leuven).

\section{RNA extraction, reverse transcription and RT-qPCR}

Total RNA was isolated using an RNeasy kit (Qiagen). $2 \mu \mathrm{g}$ of total RNA was used for first strand CDNA synthesis with SuperScript II Reverse Transcriptase and oligo-dT primers (Invitrogen). Real time PCR was performed with SYBR $\circledast$ Green PCR Master Mix (Applied Biosystems) using Quantitect primers (Qiagen) in an ABI PRISM 7900 Sequence Detection System (Applied Biosystems). The relative amount of all mRNAs was calculated using the comparative CT method after normalization to B2M and/or Bactin. 


\section{Protein analysis}

Cells were lysed in Triton lysis buffer (1\% Triton X100, 50mM Tris pH7.5, $300 \mathrm{mM} \mathrm{NaCl}, 1 \mathrm{mM}$ EGTA, 1mM DTT, 1mM NaVO4, Protease-InhibitorCocktail and Phosphatase-Inhibitor-Cocktail (Roche)). Proteins were separated on SDS-PAGE and blotted onto PVDF membrane (Immobilon). Membranes were blocked with 3\% BSA, incubated with antibody solutions and signals were detected using ECL-reagent.

\section{Generation of doxycycline-inducible shRNA cell lines and expression of p53 in SKOV3 cells}

Lentiviral vectors containing short hairpin RNA sequences targeting PFKFB4 were described previously ${ }^{35}$. shRNA sequences targeting p53 were cloned into the TetOnPLKO lentiviral vector using the following oligonucleotides: forward 5'CCGGCACCATCCACTACAACTACATCTCGAGATGTAGTTGTAGTGGATG GTGTTTTT and reverse 5'AATTAAAAACACCATCCACTACAACTACATCTCGAGATGTAGTTGTAGTG GATGGTG. Lentiviruses were produced by co-transfecting HEK 293T with shRNA plasmids and the packaging plasmids pCMV R8.91 (gag-pol) and pMD.G (VSV-G glycoprotein) ${ }^{45}$. Supernatants containing lentiviruses were collected $48 \mathrm{~h}$ after transfection, mixed with polybrene $(8 \mathrm{~g} / \mathrm{ml})$ and used for infection. Fresh medium containing puromycin $(2 \mathrm{~g} / \mathrm{ml})$ was added after 24 hours and cells were selected for at least 48 hours before use.

The coding sequence of human TP53 was excised from pCMV-Neo-Bam p53 wt (Addgene, 16434) and inserted into the lentiviral vector pBOBI (a gift from the Verma laboratory, Salk Institute, La Jolla to K. Brindle). In the resulting vector, mStrawberry is separated from TP53 by an E2A sequence (EF1-Sp53_WT), which results in expression from a single mRNA transcript in equimolar concentrations. After lentiviral transduction, SKOV3 cells displaying similar levels of fluorescence were single cell sorted using a cell sorter BD FACSAria (Flow Cytometry, CRUK-Cl), and a homogenous population was established.

\section{Chromatin immunoprecipitation (ChIP) assay}


TP53 binding sites were predicted in the promoter of the human PFKFB4 gene using the MatInspector tool from Genomatix. For ChIP assays, cells were washed with PBS and crosslinked with $1 \%$ formaldehyde for $15 \mathrm{~min}$ at $37^{\circ} \mathrm{C}$. The reaction was stopped by the addition of glycine to $125 \mathrm{mM}$ final concentration. Samples were sonicated to generate DNA fragments with an average size below 1,000 base pairs, followed by immunoprecipitation with the indicated antibodies. Bound DNA fragments were eluted and amplified by PCR using the following primer pairs: PFKFB4_p53-site1 5'CGTCCACACTGCCTGGAAA-3', 5'-CACAGCCCAACTCCATTGC-3'; PFKFB4_p53-site2 5'-TGAGCATGTGCGGAAGGA-3', 5'AAAGGAACCCATGAGGGAAGTT-3'; p21_p53-site 5'CTGAAAACAGGCAGCCCAAG-3', 5'-GTGGCTCTGATTGGCTTTCTG-3'

\section{Cell Viability Assays}

Cells were seeded on 12-well plates. After incubation, cells were fixed with $70 \%$ ethanol, stained with $0.01 \%$ crystal violet, washed and dried. For quantification, dye was extracted with $10 \%$ acetic acid and OD was measured at 560nm.

\section{Generation of PFKFB4 constructs}

The Hs_PFKFB4 cDNA was obtained from Origene and used as template for cloning into pBabe-blast using the BD In Fusion PCR Cloning kit (Clontech) and the following primers: (forward 5'TCTAGGCGCCGGCCGATGGCGTCCCAACGG and reverse 5'CTGTGCTGGCGAATTCACTGGTGAGCAGG). shRNA-insensitive constructs were generated by site-directed mutagenesis using the following primers: (PFKFB4 ${ }_{\text {ins68: }}$ forward 5'GGCCAGTATCGCCGGGATGTCGTGAACACGTATAAATCTTTTG and reverse 5'CAAAAGATTTATACGTGTTCACGACATCCCGGCGATACTGGCC;

PFKFB4 $4_{\text {ins64: }}$ forward 5'CCTGAGGTCATAGCTGCGAATATAGTCCAGGTCAAACTGGGCAGCC and reverse 5'GGCTGCCCAGTTTGACCTGGACTATATTCGCAGCTATGACCTCAGG). 


\section{Determination of Fru-2,6-BP}

Cells were homogenized in $50 \mathrm{mM} \mathrm{NaOH}$ and $0.1 \%$ Triton $\mathrm{X}-100$, heated to $80^{\circ} \mathrm{C}$ for $5 \mathrm{~min}$, and centrifuged for $5 \mathrm{~min}$. The supernatant was neutralized with acetic acid in 20mM HEPES. Fru-2,6-BP was determined as previously described ${ }^{42}$. Protein concentration was used for normalization.

\section{2-NBDG uptake}

Cells were treated with $1 \mu \mathrm{g} / \mathrm{ml}$ doxycycline for 6 days, washed with PBS and incubated in media with $100 \mu \mathrm{M}$ of 2-[N-(7-nitrobenz-2-oxa-1,3-diazol-4-yl) amino]-2-deoxy-D-glucose (2-NBDG, Sigma), a fluorescent derivative of 2doexyglucose, for 20min. Medium was removed and cells were washed twice in PBS followed by FACS analysis. DAPI was added prior to analysis to exclude dead cells.

\section{Analysis of cellular respiration}

Cells were treated with $1 \mu \mathrm{g} / \mathrm{ml}$ doxycycline for 5 days. $4 \times 10^{5}$ cells were plated in sextuplets in a 96-well XF culture plate. 24 hours later, medium was changed to assay medium (Seahorse Biosciences) supplemented with $1 \mathrm{mM}$ sodium pyruvate and $10 \mathrm{mM}$ Glucose with $\mathrm{pH}$ adjusted to 7.4. Cells were incubated in a $\mathrm{CO}_{2}$-free atmosphere for 1 hour and oxygen consumption (OCR) and extracellular acidification (ECAR) rates were determined using a XF96 Extracellular Flux Analyser (Software Version 1.4, Seahorse Biosciences, North Billerica, USA). During the experiment, $1 \mathrm{M}$ oligomycin $\mathrm{A}$ (Sigma), 0.4 M FCCP (Sigma) and 1 M antimycin A (Sigma) were injected. Rates were normalized to total protein content determined by sulforhodamine B staining.

\section{${ }^{14} \mathrm{C}$-glucose incorporation into $\mathrm{CO}_{2}$}

Cells were cultivated in the presence or absence of doxycycline for 6 days and then treated with $1 \mu \mathrm{Ci} 1-{ }^{14} \mathrm{C}$ or $6-{ }^{14} \mathrm{C}$ glucose and incubated at $37^{\circ} \mathrm{C}$ for 90min. To release ${ }^{14} \mathrm{CO}_{2}, 150 \mu \mathrm{l}$ perchloric acid was added to each well, immediately covered with phenylethylamine saturated paper and incubated at 
room temperature for $24 \mathrm{~h}$. The paper was then analysed by scintillation counting.

\section{Mass isotopomer distribution analysis}

Cells were labeled with $17.5 \mathrm{mM}$ 1,2- ${ }^{13} \mathrm{C}$-glucose (Cambridge Isotope Laboratories) for $24 \mathrm{~h}$ and extracted using acetonitrile/methanol/water (40/40/20). Dried cell extracts were resuspended in $100 \mu \mathrm{L}$ deionized water, $10 \mu \mathrm{L}$ of which were injected into a Waters Acquity UPLC with a Waters T3 column $(150 \times 2.1 \mathrm{~mm} \times 1.8 \mu \mathrm{m}$; Waters Corporation, Milford, MA, USA) coupled to a Thermo TSQ Quantum Ultra triple quadrupole instrument (Thermo Fisher Scientific, Waltham, MA, USA) with electrospray ionization. Compound separation was achieved by a gradient of two mobile phases (i) 10mM tributylamine, $15 \mathrm{mM}$ acetic acid, 5\% (v/v) methanol and (ii) 2-propanol

${ }^{3}$ and mass isotopomer distributions (MIDs) of intact and fragmented carbon backbones were acquired as described in ${ }^{37}$ and correct for natural isotopic abundance.

\section{Determination of NADPH levels}

For detection of NADPH, cells were lysed in buffer containing 20mM nicotinamide, 20mM $\mathrm{NaHCO}_{3}$ and $100 \mathrm{mM} \mathrm{Na}_{2} \mathrm{CO}_{3}$. Scraped cells were sonicated and 150 I cleared supernatants were incubated for $30 \mathrm{~min}$ at $60^{\circ} \mathrm{C}$. $20 \mu \mathrm{l}$ of heated or not heated supernatant were then mixed with $160 \mu$ I NADPcycling buffer (100mM Tris- $\mathrm{Cl}$ pH 8.0, 0.5mM thiazolyl blue, 2mM phenazine ethosulfate, 5mM EDTA and 1.3 IU glucose-6-phosphate dehydrogenase). After $1 \mathrm{~min}$ incubation in the dark at $30^{\circ} \mathrm{C}, 1 \mathrm{mM}$ glucose-6-phosphate was added and the change in absorbance at 570nm was measured every $30 \mathrm{sec}$ for $4 \mathrm{~min}$ at $30^{\circ} \mathrm{C}$. Protein concentration was used for normalization.

\section{Determination of acetate-dependent lipid synthesis}

Cells were incubated in medium containing $10 \mu \mathrm{Ci} / \mathrm{ml}\left[1-{ }^{14} \mathrm{C}\right]$ acetate $(85 \mathrm{M}$ final concentration, Perkin Elmer) for 3 hours. After washing three times with PBS, cells were lysed in 0.5\% Triton X-100/PBS. Lipids were extracted by successive addition of $2 \mathrm{ml}$ methanol, $2 \mathrm{ml}$ chloroform, and $1 \mathrm{ml} \mathrm{dH}_{2} \mathrm{O}$. Phases were separated by centrifugation before the organic phase was dried and 
used for scintillation counting. Results were normalized to total protein content determined by sulforhodamine B staining.

\section{Reporter assays}

Cells were transfected with 0.5 $\mu$ g pGL3-SYNwt-luc or pGL3-SYNmutSRE1 41 and 0.5ng phRL-SV40 using Lipofectamine ${ }^{\mathrm{TM}}$ 2000. Cells were lysed and activity was determined using a Dual Luciferase assay (Promega).

\section{Determination of ROS levels}

ROS levels were determined by incubating cells for $30 \mathrm{~min}$ at $37^{\circ} \mathrm{C}$ in medium with $1.6 \mu \mathrm{M} \mathrm{CM}-\mathrm{H}_{2}$-DCFDA (Molecular Probes) followed by FACS analysis. DAPI was added prior to analysis to exclude dead cells.

\section{Apoptosis assay}

Cells were trypsinised and stained with Annexin V-pacific blue and propidium iodide. Apoptotic cells were determined by FACS analysis.

\section{Induction of spheroid growth}

For spheroid formation, cells were trypsinized, counted and placed in 96-well ultralow attachment plates (Costar). Spheroid formation was initiated by centrifugation at $650 \times \mathrm{g}$ for $10 \mathrm{~min}$ and cultures were incubated for 13 days. Spheroid size was determined by imaging on an inverted microscope (Axiovert 100M, Carl Zeiss) and images were processed in ImageJ.

\section{Xenograft experiments}

To generate luciferase expressing cells, a pBabe-Luciferase retroviral vector (a gift from J. Downward) was packaged in Phoenix-Ampho cells and used to

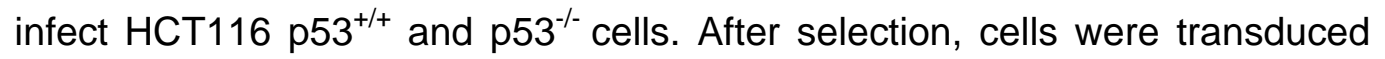
with lentiviral vectors and selected as in Generation of doxycycline-inducible shRNA cell lines section. $1 \times 10^{6}$ cells were subcutaneously injected into the dorsal flank of male nude mice (ICRF nude). Animals were randomly assigned to two cohorts. For induction of shRNA expression, one animal cohort received doxycycline (Doxycycline diet, $0.2 \mathrm{~g} / \mathrm{kg}$ food pellet, Harlan D.98186) starting 1 day after implantation, and tumour growth was followed over 21 
days. HCT116 luc $53^{+/+}$shCrtl $(n=6)$, HCT116 luc $553^{+/+}$shCtrl DOX $(n=6)$, HCT116 luc $553^{+/+}$shB4\#68 (n=5), HCT116 luc p53 ${ }^{+/+}$shB4\#68 DOX $(n=4)$, HCT116 luc p53 ${ }^{-/}$shCtrl (n=5), HCT116 luc p53 ${ }^{-/}$shCtrl DOX $(n=6)$, HCT116 luc p53l-shB4\#68 (n=4), HCT116 luc shB4\#68 DOX (n=6), HCT116 luc p53 ${ }^{+/+}$shB4\#64 $(n=6)$, HCT116 luc p53 ${ }^{+/+}$shB4\#64 DOX $(n=5)$, HCT116 luc p53 $3^{-/-}$shB4\#68 (n=5), HCT116 luc shB4\#68 DOX ( $n=5)$. Mice were anaesthetised, intraperitoneally injected with Luciferin (Promega) and imaged using an IVIS Spectrum imaging system. Investigators were blinded to group allocation. Images were analysed using the IVIS Living Image software. Criteria for the exclusion of animal from the analysis were pre-defined based on morbidity unrelated to experimental treatment. This was not observed during the experiment and no animals were excluded. All animal experiments were performed according to UK Home Office guidelines.

\section{Histology}

$4 \mathrm{~m}$ thick tissue sections were mounted, dewaxed, and rehydrated. Antigen retrieval was performed with citrate buffer $(\mathrm{pH}$ 6.0) in a microwave oven for 30min. Endogenous peroxidase activity was blocked by incubating sections in $3 \%(\mathrm{v} / \mathrm{v})$ hydrogen peroxide for $10 \mathrm{~min}$. Sections were blocked with 3\% (w/v) BSA for 30min and incubated with the monoclonal mouse anti-human Ki-67 antibody (9106, Thermo Scientific), diluted 1:200 over night at $4^{\circ} \mathrm{C}$ in a humidified chamber. Sections were washed in PBS and incubated with biotinylated secondary antibody followed by streptavidin-horseradishperoxidase assay (Vector Labs, Dako). Reaction was developed using 3,3'diaminobenzidine (Cell Signaling). Slides were counterstained with Gilmore 3 hematoxylin, dehydrated, cleared, and mounted with coverslips. TUNEL assay was performed according to the manufacturer's instructions (In Situ Cell Death Detection Kit, Roche no. 11684795910).

\section{RNAseq analysis}

Total RNA from 3 tumours in each treatment group (HCT116 luc $553^{+/+}$shCtrl, HCT116 luc p53 ${ }^{+/+}$shB4\#68, HCT116 luc $53^{-{ }^{-}}$shCtrl and HCT116 luc p53 ${ }^{- \text {shB }^{-}}$sh68 all treated with DOX) was extracted using RNeasy mini columns (Qiagen) with on column DNase I digestion. PolyA+ RNA was extracted using the NEBNext 
Poly(A) mRNA Magnetic Isolation Module (NEB, Ipswich, MA, USA). For library preparation, the NEBNext Ultra RNA Library Prep Kit for Illumina (NEB, Ipswich, MA, USA) was used. Quantification and quality control was performed using the Experion Automated Electrophoresis System (BioRad, Hercules, CA, USA). Sequencing was performed using an Illumina NextSeq500 System (Illumina, San Diego, CA, USA).

Fastq files were mapped to the human genome assembly hg19 using TopHat v2.0.7 with default parameters and subsequently normalized to the number of mapped reads in the smallest sample. Reads per gene were counted using the countOverlaps function from the $\mathrm{R}$ package (Genomic Ranges). Weakly expressed genes were removed (threshold: mean read count over all samples $<1)$ and differentially expressed genes were called using EdgeR. GSE analyses ${ }^{40}$ were performed with signal2noise metric, 1000 permutations and the C2 gene set. Pathway analysis of differentially expressed genes (logFC 0.7, q 0.05) was performed using the DAVID tool ${ }^{14,15}$ with default settings.

\section{Analysis of patient data and survival analysis}

Lung cancer data were downloaded from the Gene Expression Omnibus (GSE12667) and MAS 5.0 processed signals were generated on Affymetrix Human Genome U133 Plus 2.0. Normalised data were log2 transformed. A Wilcoxon rank sum test was used to assess significant chances in expression

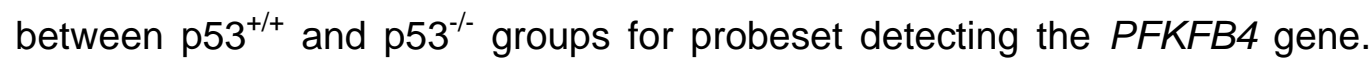
Analysis of survival data from public datasets for breast cancer ${ }^{16}$ and nonsmall cell lung cancer ${ }^{24}$ was performed using PROGene ${ }^{11}$. Patients were divided based on median PFKFB4 expression and relapse-free survival was analysed. Expression data for PFKFB4 in lung adenocarcinoma were extracted from public datasets ${ }^{8}$.

\section{Statistical analysis}

Graphs were generated using GraphPad Prism 5.0 (GraphPad software). All experiments were performed with three biologically independent replicates unless stated otherwise. Sample sizes are calculated to allow significance to be reached. Statistical significance of magnitude of changes between different 
conditions was calculated using the parametric two-tailed unpaired Student ttests. Statistical significance was defined as a $p$-value of less than 0.05. $P$ values are depicted as follows: ${ }^{\star} p$ 0.05, ${ }^{* \star} p$ 0.01, ${ }^{* \star *} p 0.001$ and ${ }^{\star \star \star \star} p$ 0.0001. n.s. = not significant.

\section{CONFLICT OF INTEREST}

The authors declare no conflict of interest. Correspondence and requests for materials should be addressed to A.S. (almut.schulze@uni-wuerzburg.de).

\section{ACKNOWLEDGEMENTS}

We thank C. Esnault (LRI) for advice on ChIP and $\mathrm{H}$. Miess for help with Seahorse Analysis, C. Watkins and J. Bee (LRI, BRU) for help with animal experimentation, F. Lassailly, P. Johnson and T. Snoeks (In vivo imaging facility, LRI) for assistance with in vivo imaging and the LRI research services for technical support. We also thank C. Ade and Barbara Bauer (TheodorBoveri-Institute, Würzburg) for help with RNAseq analysis and histology, Beatrice Dankworth for help with cell line generation and W. Schmitz for insightful discussions. Cancer Research UK, the German Cancer Aid (FOR 2314), and the CRUK-EPSRC Imaging Centre in Cambridge and Manchester grant (16465) supported this work.

\section{AUTHOR CONTRIBUTION}

S.R. and A.S. conceived the project and wrote the manuscript. S.R. J.F., I.K., C.D., A.H., S.D., B.G and S.B. performed experiments and analysis of results. R.M. and S.W performed the bioinformatic analysis. A.B., K.M.B, N.Z., M.H.R. contributed to the study design and data analysis. All authors commented on the manuscript. 


\section{REFERENCES}

1 Bengoechea-Alonso MT, Ericsson J. SREBP in signal transduction: cholesterol metabolism and beyond. Curr Opin Cell Biol 2007; 19: 215222.

2 Bensaad K, Tsuruta A, Selak MA, Vidal MN, Nakano K, Bartrons R et al. TIGAR, a p53-inducible regulator of glycolysis and apoptosis. Cell 2006; 126: 107-120.

3 Buescher JM, Moco S, Sauer U, Zamboni N. Ultrahigh performance liquid chromatography-tandem mass spectrometry method for fast and robust quantification of anionic and aromatic metabolites. Analytical chemistry 2010; 82: 4403-4412.

4 Bunz F, Dutriaux A, Lengauer C, Waldman T, Zhou S, Brown JP et al. Requirement for p53 and p21 to sustain G2 arrest after DNA damage. Science 1998; 282: 1497-1501.

5 Casciari JJ, Sotirchos SV, Sutherland RM. Variations in tumor cell growth rates and metabolism with oxygen concentration, glucose concentration, and extracellular $\mathrm{pH}$. Journal of cellular physiology 1992; 151: 386-394.

6 Chesney J, Clark J, Klarer AC, Imbert-Fernandez Y, Lane AN, Telang S. Fructose-2,6-bisphosphate synthesis by 6-phosphofructo-2kinase/fructose-2,6-bisphosphatase 4 (PFKFB4) is required for the glycolytic response to hypoxia and tumor growth. Oncotarget 2014; 5: 6670-6686.

7 Denko NC. Hypoxia, HIF1 and glucose metabolism in the solid tumour. Nat Rev Cancer 2008; 8: 705-713.

8 Ding L, Getz G, Wheeler DA, Mardis ER, McLellan MD, Cibulskis K et al. Somatic mutations affect key pathways in lung adenocarcinoma. Nature 2008; 455: 1069-1075.

9 Feng Z, Hu W, de Stanchina E, Teresky AK, Jin S, Lowe S et al. The regulation of AMPK beta1, TSC2, and PTEN expression by p53: stress, cell and tissue specificity, and the role of these gene products in modulating the IGF-1-AKT-mTOR pathways. Cancer Res 2007; 67: 3043-3053.

10 Freed-Pastor WA, Mizuno $\mathrm{H}$, Zhao X, Langerod A, Moon SH, Rodriguez-Barrueco $\mathrm{R}$ et al. Mutant p53 Disrupts Mammary Tissue Architecture via the Mevalonate Pathway. Cell 2012; 148: 244-258.

11 Goswami CP, Nakshatri H. PROGgene: gene expression based survival analysis web application for multiple cancers. Journal of clinical bioinformatics 2013; 3: 22. 
12 Hitosugi $\mathrm{T}$, Zhou L, Elf $\mathrm{S}$, Fan J, Kang HB, Seo JH et al. Phosphoglycerate mutase 1 coordinates glycolysis and biosynthesis to promote tumor growth. Cancer cell 2012; 22: 585-600.

13 Ho J, Benchimol S. Transcriptional repression mediated by the p53 tumour suppressor. Cell death and differentiation 2003; 10: 404-408.

14 Huang da W, Sherman BT, Lempicki RA. Systematic and integrative analysis of large gene lists using DAVID bioinformatics resources. Nat Protoc 2009; 4: 44-57.

15 Huang DW, Sherman BT, Tan Q, Kir J, Liu D, Bryant D et al. DAVID Bioinformatics Resources: expanded annotation database and novel algorithms to better extract biology from large gene lists. Nucleic Acids Res 2007; 35: W169-175.

16 Ivshina AV, George J, Senko O, Mow B, Putti TC, Smeds J et al. Genetic reclassification of histologic grade delineates new clinical subtypes of breast cancer. Cancer research 2006; 66: 10292-10301.

17 Jiang P, Du W, Wang X, Mancuso A, Gao X, Wu M et al. p53 regulates biosynthesis through direct inactivation of glucose-6-phosphate dehydrogenase. Nature cell biology 2011; 13: 310-316.

18 Jiang P, Du W, Mancuso A, Wellen KE, Yang X. Reciprocal regulation of p53 and malic enzymes modulates metabolism and senescence. Nature 2013; 493: 689-693.

19 Kawauchi K, Araki K, Tobiume K, Tanaka N. p53 regulates glucose metabolism through an IKK-NF-kappaB pathway and inhibits cell transformation. Nat Cell Biol 2008; 10: 611-618.

20 Kim HR, Roe JS, Lee JE, Cho EJ, Youn HD. p53 regulates glucose metabolism by miR-34a. Biochem Biophys Res Commun 2013; 437: 225-231.

21 Kruiswijk F, Labuschagne CF, Vousden KH. p53 in survival, death and metabolic health: a lifeguard with a licence to kill. Nature reviews Molecular cell biology 2015; 16: 393-405.

22 Laplante $M$, Sabatini DM. An emerging role of $\mathrm{mTOR}$ in lipid biosynthesis. Curr Biol 2009; 19: R1046-1052.

23 Lavin MF, Gueven N. The complexity of p53 stabilization and activation. Cell death and differentiation 2006; 13: 941-950.

24 Lee ES, Son DS, Kim SH, Lee J, Jo J, Han J et al. Prediction of recurrence-free survival in postoperative non-small cell lung cancer patients by using an integrated model of clinical information and gene expression. Clin Cancer Res 2008; 14: 7397-7404. 
25 Li T, Kon N, Jiang L, Tan M, Ludwig T, Zhao $\mathrm{Y}$ et al. Tumor Suppression in the Absence of p53-Mediated Cell-Cycle Arrest, Apoptosis, and Senescence. Cell 2012; 149: 1269-1283.

26 Lunt SY, Vander Heiden MG. Aerobic glycolysis: meeting the metabolic requirements of cell proliferation. Annu Rev Cell Dev Biol 2011; 27: 441-464.

27 Maddocks OD, Vousden $\mathrm{KH}$. Metabolic regulation by p53. J Mol Med (Berl) 2011; 89: 237-245.

28 Maddocks OD, Berkers CR, Mason SM, Zheng L, Blyth K, Gottlieb E et al. Serine starvation induces stress and p53-dependent metabolic remodelling in cancer cells. Nature 2013; 493: 542-546.

29 Matoba S, Kang JG, Patino WD, Wragg A, Boehm M, Gavrilova O et al. p53 Regulates Mitochondrial Respiration. Science 2006.

30 Mayers JR, Vander Heiden MG. Famine versus feast: understanding the metabolism of tumors in vivo. Trends Biochem Sci 2015.

31 Murphy M, Ahn J, Walker KK, Hoffman WH, Evans RM, Levine AJ et al. Transcriptional repression by wild-type p53 utilizes histone deacetylases, mediated by interaction with mSin3a. Genes \& development 1999; 13: 2490-2501.

32 Okar DA, Manzano A, Navarro-Sabate A, Riera L, Bartrons R, Lange AJ. PFK-2/FBPase-2: maker and breaker of the essential biofactor fructose-2,6-bisphosphate. Trends Biochem Sci 2001; 26: 30-35.

33 Ralser M, Wamelink MM, Kowald A, Gerisch B, Heeren G, Struys EA et al. Dynamic rerouting of the carbohydrate flux is key to counteracting oxidative stress. J Biol 2007; 6: 10.

34 Rinn JL, Huarte M. To repress or not to repress: this is the guardian's question. Trends Cell Biol 2011; 21: 344-353.

35 Ros S, Santos CR, Moco S, Baenke F, Kelly G, Howell M et al. Functional metabolic screen identifies 6-phosphofructo-2kinase/fructose-2,6-biphosphatase 4 as an important regulator of prostate cancer cell survival. Cancer Discov 2012; 2: 328-343.

36 Ros S, Schulze A. Balancing glycolytic flux: the role of 6phosphofructo-2-kinase/fructose 2,6-bisphosphatases in cancer metabolism. Cancer Metab 2013; 1: 8.

37 Ruhl M, Rupp B, Noh K, Wiechert W, Sauer U, Zamboni N. Collisional fragmentation of central carbon metabolites in LC-MS/MS increases 
precision of (1)(3)C metabolic flux analysis. Biotechnol Bioeng 2012; 109: 763-771.

38 Schwartzenberg-Bar-Yoseph F, Armoni M, Karnieli E. The tumor suppressor p53 down-regulates glucose transporters GLUT1 and GLUT4 gene expression. Cancer research 2004; 64: 2627-2633.

39 Strohecker AM, Joshi S, Possemato R, Abraham RT, Sabatini DM, White E. Identification of 6-phosphofructo-2-kinase/fructose-2,6bisphosphatase as a novel autophagy regulator by high content shRNA screening. Oncogene 2015.

40 Subramanian A, Tamayo P, Mootha VK, Mukherjee S, Ebert BL, Gillette MA et al. Gene set enrichment analysis: a knowledge-based approach for interpreting genome-wide expression profiles. Proceedings of the National Academy of Sciences of the United States of America 2005; 102: 15545-15550.

41 Swinnen JV, Heemers H, Deboel L, Foufelle F, Heyns W, Verhoeven G. Stimulation of tumor-associated fatty acid synthase expression by growth factor activation of the sterol regulatory element-binding protein pathway. Oncogene 2000; 19: 5173-5181.

42 Van Schaftingen E, Lederer B, Bartrons R, Hers HG. A kinetic study of pyrophosphate: fructose-6-phosphate phosphotransferase from potato tubers. Application to a microassay of fructose 2,6-bisphosphate. European journal of biochemistry / FEBS 1982; 129: 191-195.

43 Willis A, Jung EJ, Wakefield T, Chen X. Mutant p53 exerts a dominant negative effect by preventing wild-type p53 from binding to the promoter of its target genes. Oncogene 2004; 23: 2330-2338.

44 Yahagi N, Shimano H, Matsuzaka T, Najima Y, Sekiya M, Nakagawa Y et al. p53 Activation in adipocytes of obese mice. The Journal of biological chemistry 2003; 278: 25395-25400.

45 Zufferey R, Nagy D, Mandel RJ, Naldini L, Trono D. Multiply attenuated lentiviral vector achieves efficient gene delivery in vivo. Nature biotechnology 1997; 15: 871-875. 


\section{FIGURE LEGENDS}

\section{Figure 1: Differential expression of PFKFB4 in p53 deficient cells}

a) HCT116 $\mathrm{p} 53^{+/+}$or $\mathrm{p} 53^{-/-}$cells were cultured in full medium for 24 hours. Expression of PFKFB1, PFKFB2, PFKFB3 and PFKFB4 was determined by qPCR and normalised to B2M. Data are presented as mean \pm SEM $(n=6)$.

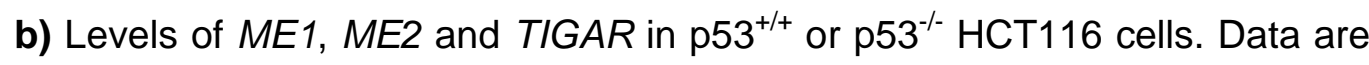
presented as mean \pm SEM $(n=6)$.

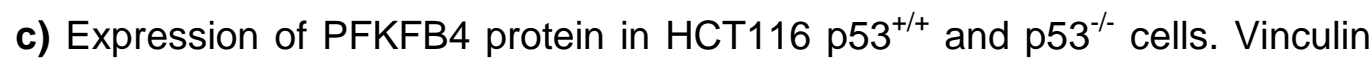
was used as loading control. Bar graph shows the mean \pm SEM of 3 replicate experiments.

d) MEFs from tpr53 $3^{f / f l}$ mice were infected with Adeno-CRE-GFP to excise the floxed allele. mRNA from selected cells was analysed for expression of Pfkfb4. Data are presented as mean \pm SEM (tpr53+/+ n=7 wt, tpr53-/- n=8).

e) HCT116 ${\mathrm{p} 53^{+/+}}^{+/ l}$ cels were transduced with lentiviral vectors expressing inducible shRNA targeting p53. Cells were treated with $1 \mu \mathrm{g} / \mathrm{ml}$ doxycycline (Dox) or solvent for 8 days. Expression of PFKFB4, ME2, TP53 and p21 was determined by qPCR. Data are presented as mean \pm SEM $(n=4)$.

f) SKOV3 cells were transduced with a lentiviral vector expressing wild type p53 or a control vector. Expression of TP53, PFKFB4 and ME2 was determined by qPCR in monoclonal populations. Data are presented as mean $\pm \operatorname{SD}(n=3)$.

g) Expression of PFKFB4 and p53 protein in cells as F. Actin was used as loading control.

\section{Figure 2: p53 represses PFKFB4 expression in cancer cells}

a) $\mathrm{HCT} 116{\mathrm{p} 53^{+/+}}$cells were treated with different concentrations of etoposide for 24 hours. Expression of PFKFB4, p21 (CDKN1A) and ME2 was determined by qPCR. Data are presented as mean \pm SEM $(n=3)$.

b) Regulation of p21, PFKFB4 and ME2 in p53 wild type LOVO colon cancer cells after etoposide treatment. Data are presented as mean $\pm S D(n=3)$.

c) Regulation of p21, PFKFB4 and ME2 in p53 wild type MCF7 breast cancer cells after etoposide treatment. Data are presented as mean $\pm S D(n=3)$.

d) Overview of the structure of the human PFKFB4 promoter. Sequences corresponding to putative p53 response elements are indicated. (B4 site 1: 
position chr3:48598858-48598880; B4-site 2: position: chr3:48592906-

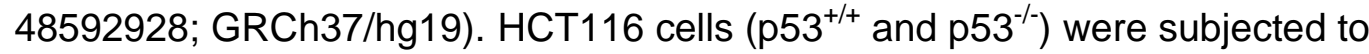
chromatin immunoprecipitation assays using p53 specific antibodies (TP53 $A b)$, isotype-matched controls (IgG) or no antibody (no Ab). Promoter regions corresponding to p53-site 1 (left panel) or p53-site2 (right panel) of the PFKFB4 promoter were amplified using qPCR. A p53-site in the p21 promoter was used as positive control.

e) HCT116 p53 ${ }^{+/+}$cells, LOVO and MCF7 cancer cells were treated with $20 \mu \mathrm{M}$ etoposide in the presence or absence of 100nM TSA. Expression of p21, PFKFB4 and ME2 mRNA was determined by qPCR. HCT $\mathrm{p} 53^{+/+}$cells data are presented as mean \pm SEM $(n=2)$. LOVO and MCF7 data are presented as mean $\pm S D(n=3)$.

Figure 3: PFKFB4 is required for viability of p53 deficient cancer cells

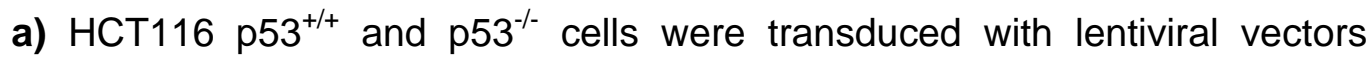
expressing inducible shRNA targeting PFKFB4 (shPFKFB4 \#68 or \#64). Cells were seeded at low density, treated with $1 \mu \mathrm{g} / \mathrm{ml}$ doxycycline (Dox) or solvent for 8 days and stained with crystal violet. Left: representative images. Right: Quantification. Data are presented as mean \pm SEM $(n=5)$ and are relative to

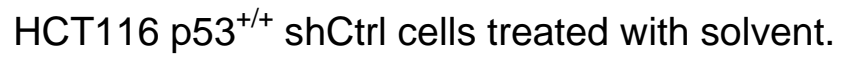

b) Cells were treated with $1 \mu \mathrm{g} / \mathrm{ml}$ doxycycline (Dox) or solvent for 6 days and expression of PFKFB4 was determined by qPCR and normalised to B2M. Data are presented as mean \pm SEM $(n=5)$ and are relative to HCT116 p53 ${ }^{+/+}$ shPFKFB4 cells treated with solvent.

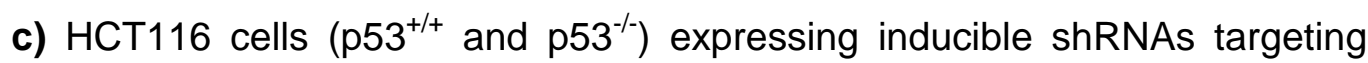
PFKFB4 were stably transfected with pBabe-vectors expressing shRNAinsensitive versions of PFKFB4 (PFKFB4 ${ }_{\text {ins }}$ ). Cells were seeded al low density and treated with doxycycline (Dox) for 8 days. Cultures were fixed and analysed by crystal violet staining. Data are presented as mean \pm SEM $(n=6)$. d) Expression of PFKFB4 in p53 wild type cells (RKO, LOVO, LS174T) or p53 mutant cells (SW480 and SW620) was determined by qPCR relative to B2M. Box plot represents the differential expression of PFKFB4 between both groups of cell lines. 
e) LOVO, LS174Tm SW480 and SW460 cells were transduced with lentiviral vectors expressing inducible shRNA targeting PFKFB4 (shPFKFB4 \#68 or \#64). Silencing of PFKFB4 was confirmed by qPCR.

f) Cells in $E$ were seeded at low density, treated with $1 \mu \mathrm{g} / \mathrm{ml}$ doxycycline (Dox) or solvent for 8 days and stained with crystal violet. Data are presented as mean $\pm \operatorname{SEM}(n=3)$ and are relative to cells treated with solvent.

Figure 4: PFKFB4 maintains balance between glycolysis and pentose phosphate pathway in p53 deficient cancer cells

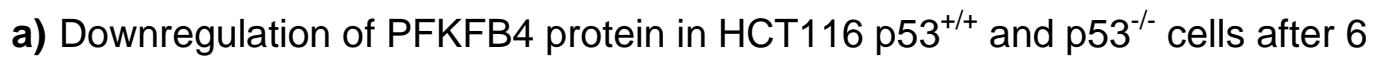
days of shRNA-mediated silencing. Actin was used as loading control.

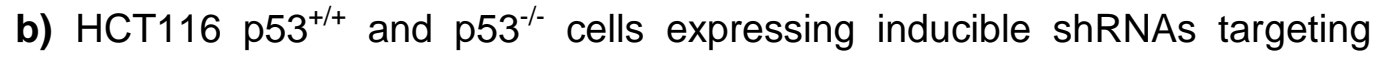
PFKFB4 were treated with $1 \mu \mathrm{g} / \mathrm{ml}$ doxycycline (Dox) or solvent for 6 days. Intracellular levels of fructose 2,6-biphosphate (Fru-2,6-BP) were determined. Data are presented as mean $\pm \operatorname{SEM}(n=5)$.

c) Extracellular acidification rates (ECAR) and oxygen consumption rates (OCR) were determined in cells treated as in A. Graph displays ECAR/OCR and data are presented as mean \pm SEM.

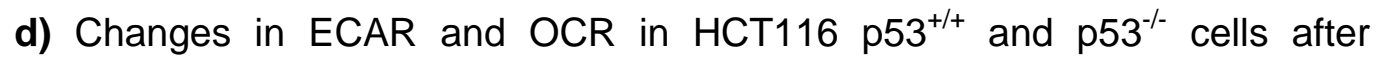
silencing of PFKFB4. Data are presented as mean \pm SEM $(n=5)$.

e) HCT116 p53 ${ }^{+/+}$and $p 53^{-/-}$cells were treated with doxycycline (Dox) for 6 days and the labelled with either ${ }^{14} \mathrm{C}_{1}$ or ${ }^{14} \mathrm{C}_{6}$ glucose. Relative production of $\mathrm{CO}_{2}$ from both substrates was determined. Data are presented as mean \pm $\operatorname{SEM}(n=4)$.

Figure 5: PFKFB4 maintains NADPH production for biosynthetic activity and antioxidant production in p53 deficient cancer cells

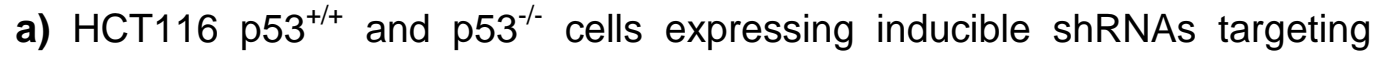
PFKFB4 were treated with $1 \mu \mathrm{g} / \mathrm{ml}$ doxycycline (Dox) or solvent for 6 days. Levels of NADP+ and NADPH were determined and normalised to total protein content. Values are presented relative to solvent controls. Data are presented as mean $\pm \operatorname{SEM}(n=8)$.

b) Rates of acetate-dependent lipid biosynthesis in HCT116 $\mathrm{p} 53^{+/+}$and $\mathrm{p} 53^{-/-}$ cells. Data are presented as mean $\pm \operatorname{SEM}(n=6)$. 


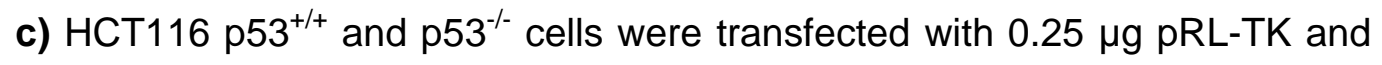
$2.5 \mu \mathrm{g}$ pGL3-SYNwt-luc (SYN-wt) or pGL3-SYNmutSRE1 (SYN-mutSRE1). Firefly luciferase activity was determined 48 hours post-transfection and normalised to renilla luciferase. Data are presented as mean $\pm S D(n=3)$.

d) Rates of acetate-dependent lipid biosynthesis in HCT116 p53 ${ }^{+/+}$and p53 $3^{-/-}$ cells after 6 days of PFKFB4 silencing. Data are presented as mean \pm SD $(n=3)$.

e) HCT116 ( $\mathrm{p} 53^{+/+}$and $\mathrm{p53}^{-{ }^{--}}$) cells expressing shRNAs targeting PFKFB4 or controls were treated with $1 \mu \mathrm{g} / \mathrm{ml}$ doxycycline (Dox) for 6 days. ROS levels were determined by DCFDA fluorescence. Data are presented as mean \pm $\operatorname{SEM}(n=3)$.

f) HCT116 p53-/- cells were depleted of PFKFB4 as in E. Cells were treated with 10mM N-acetyl cysteine (NAC) 24 hours prior to detection of ROS $(n=5)$.

g) Diagram of allosteric regulation of glycolytic flux and pentose phosphate pathway (PPP) activity by PFKFB4.

\section{Figure 6: PFKFB4 supports tumour growth in p53 deficient cancer cells}

a) $\mathrm{HCT}_{116}$ luc $_{\mathrm{p}} 53^{+/+}$and $\mathrm{p53-/-}$ cells expressing inducible shRNAs targeting PFKFB4 (shPFKB4\#68 above and shPFKFB4 \#64 below) were injected subcutaneously into nude mice. Mice were divided into two cohorts with equal tumour burden and one cohort received doxycycline in their food (DOX) started one day after implantation. Tumour growth was monitored by in vivo quantification of luciferase. Graphs show mean bioluminescence (p/sec/cm2/sr) \pm SEM for each group.

b) Representative mice for each group at day 21 are shown.

c) Analysis of fragmented DNA in apoptotic cells using TUNEL assays in tumours recovered at day 21 , representative images.

d) Analysis of proliferation using histological staining for the Ki67 marker in tumours recovered at day 21, representative images.

e) Selected gene sets showing decreased expression in tumours depleted of PFKFB4 compared to controls by gene set enrichment analysis (GSEA). 


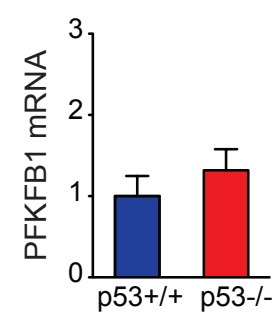

b
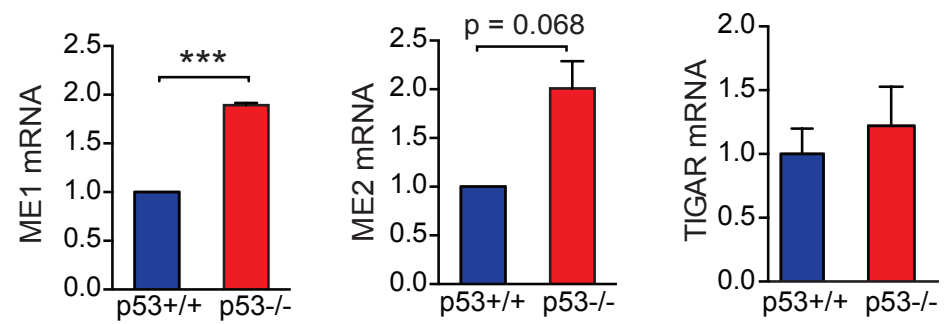

d
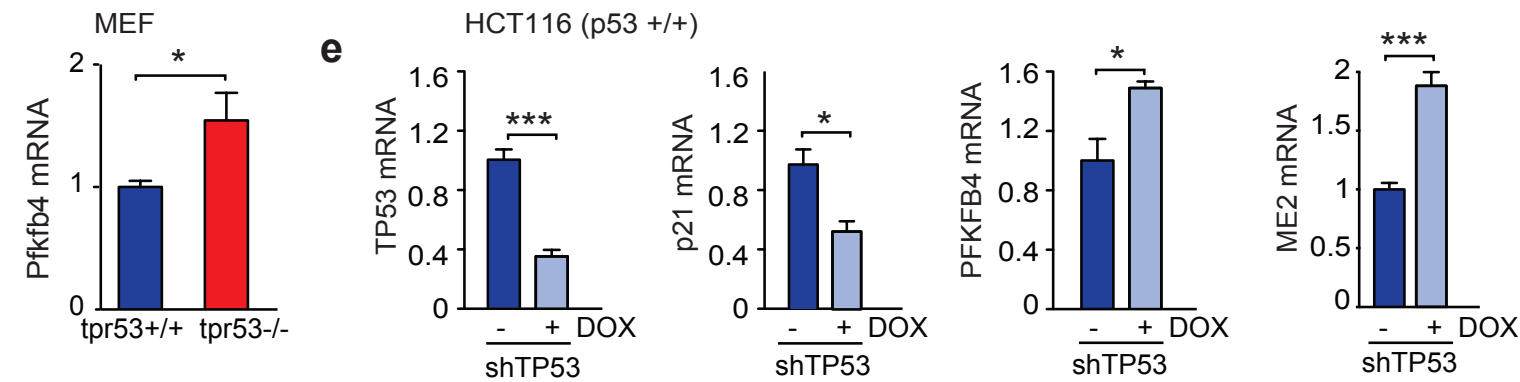

f
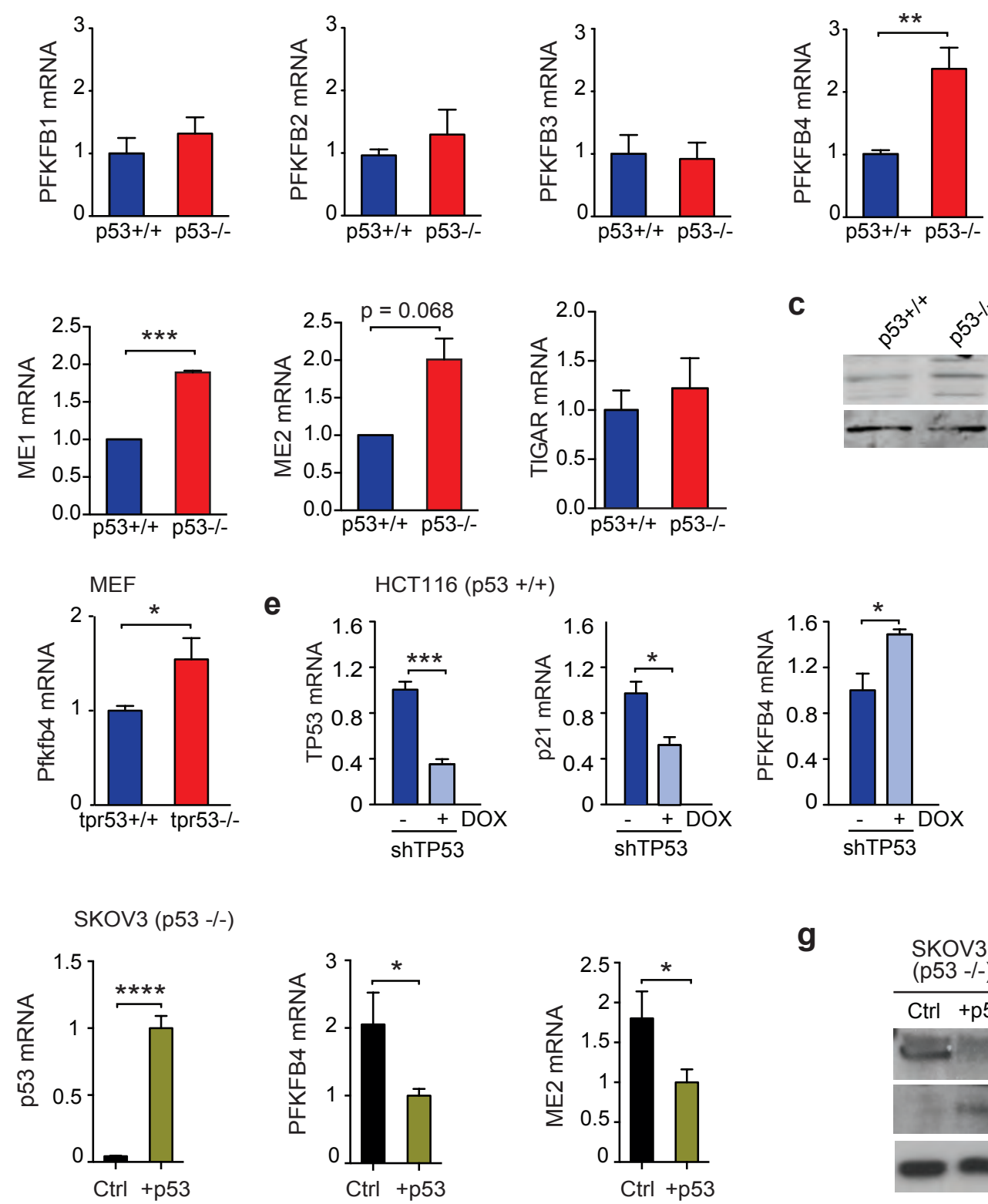

C
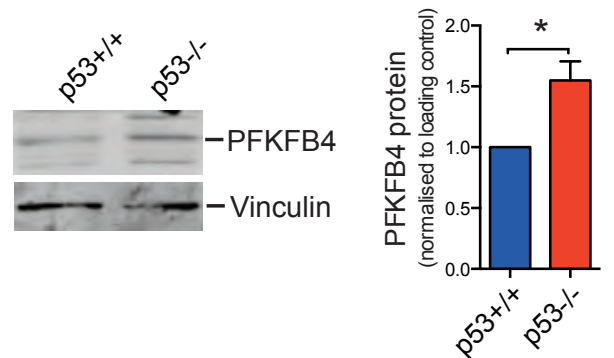

g

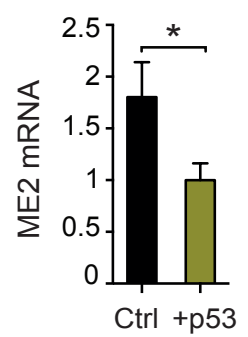

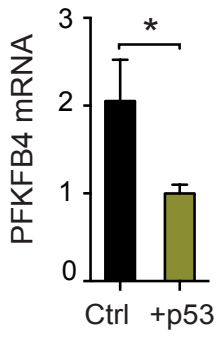

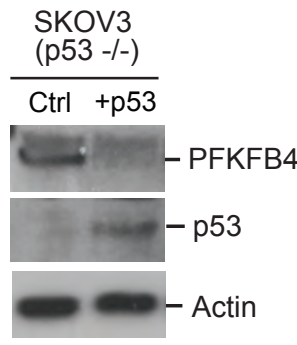


HCT $($ p53 +/+)
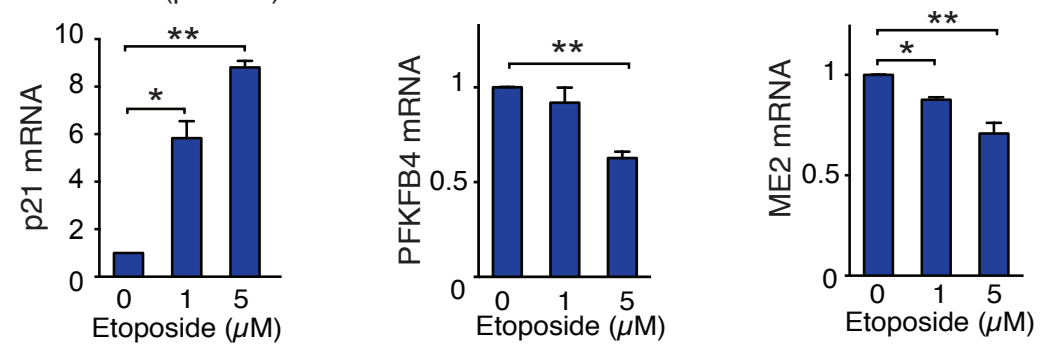

Ros et al. Figure 2

b LOVO (p53 WT)
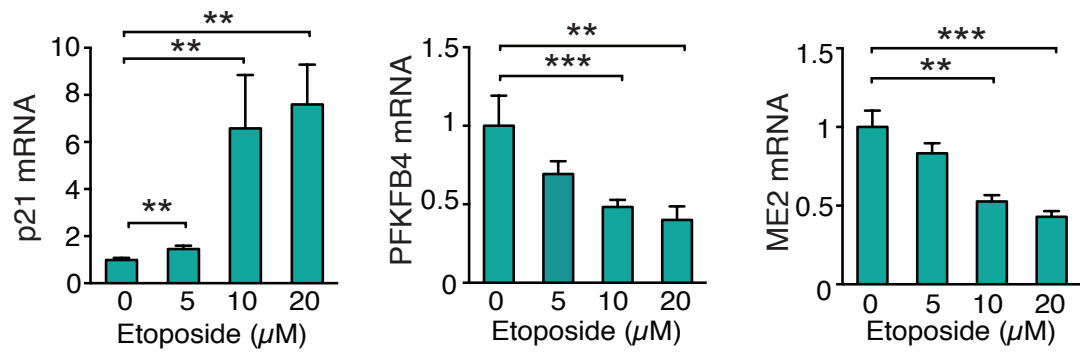

C
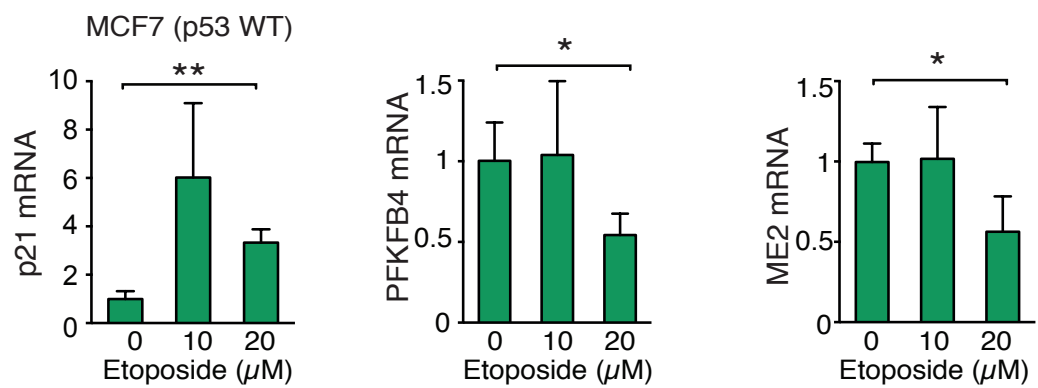

d
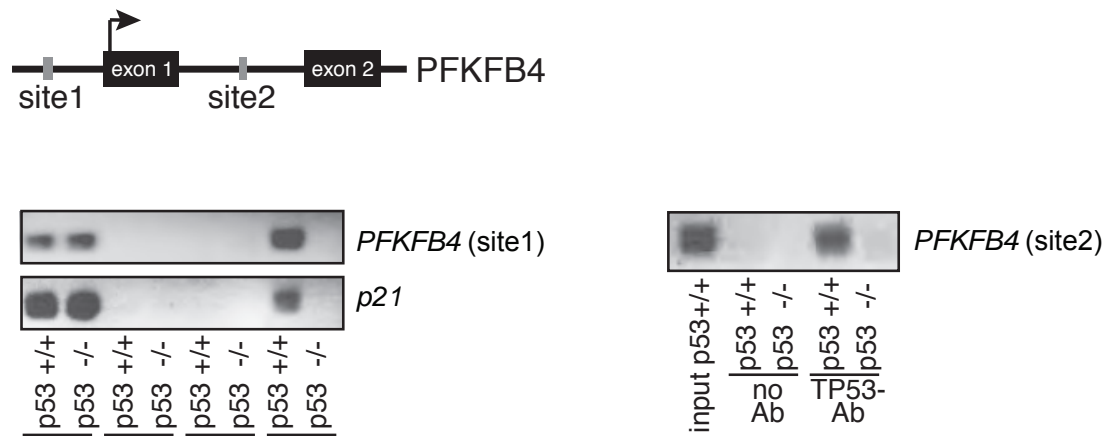

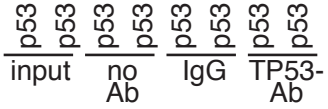

e
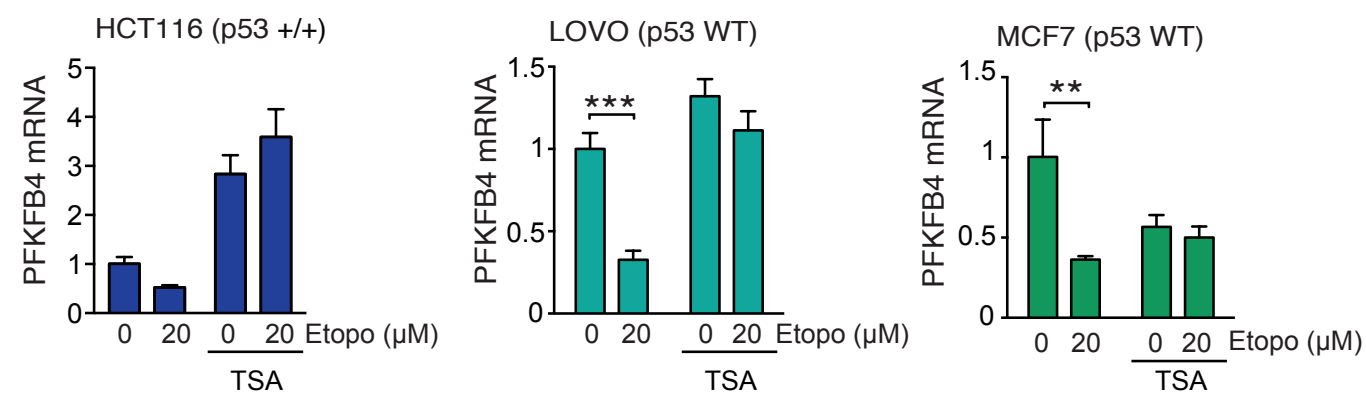

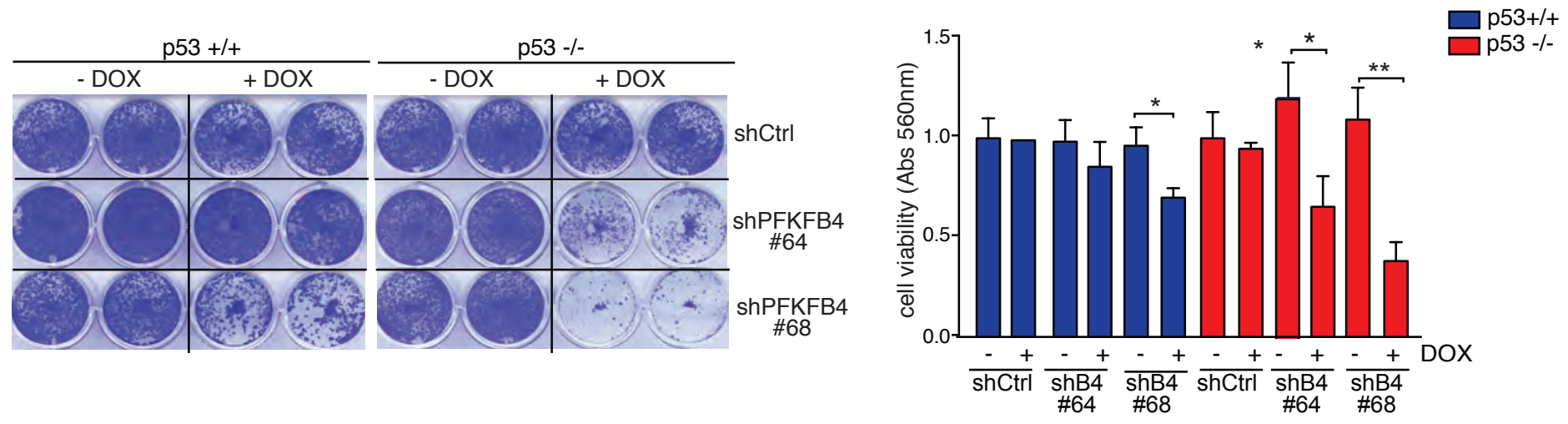

b

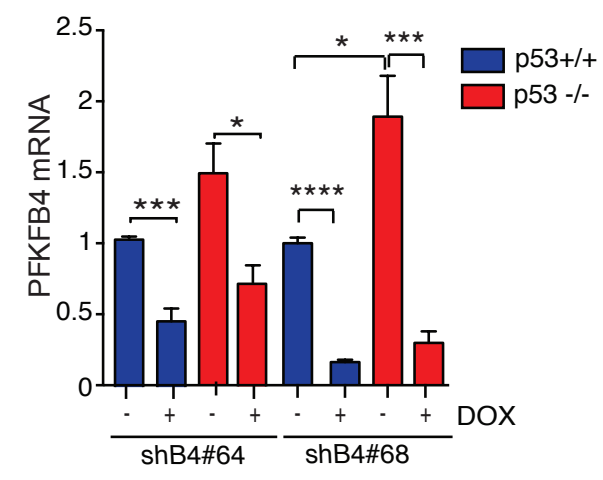

C

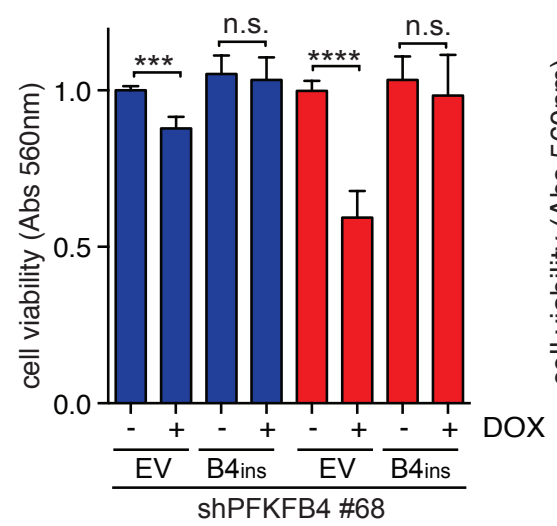

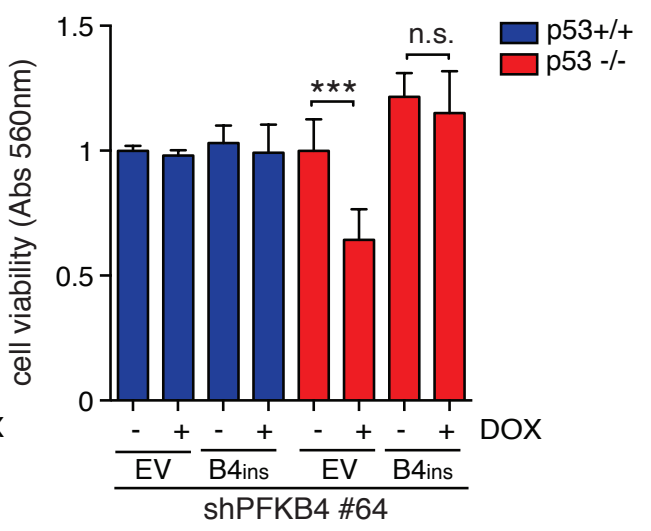

d

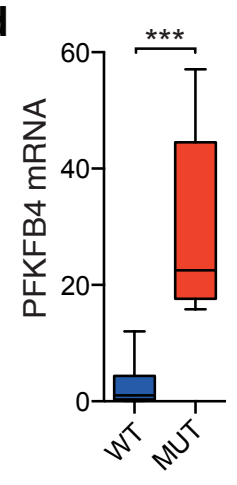

e

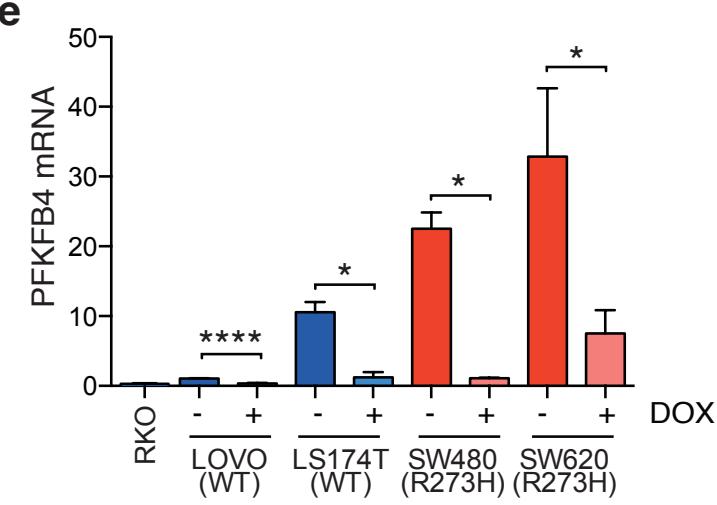

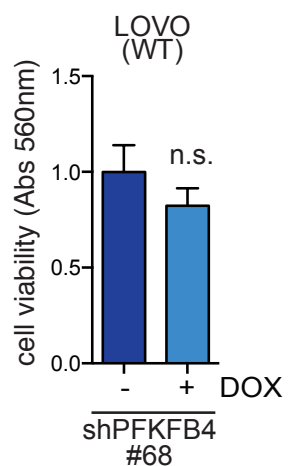
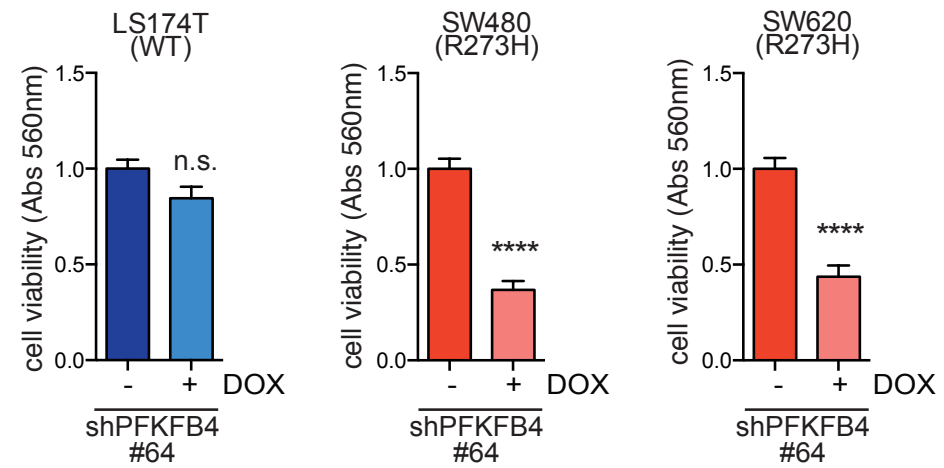
Ros et al. Figure 4

a

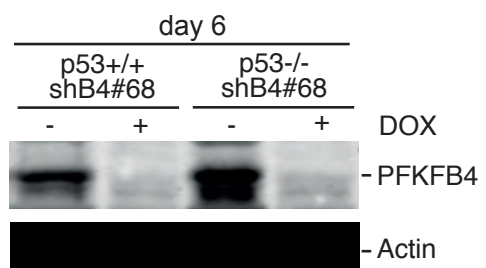

d

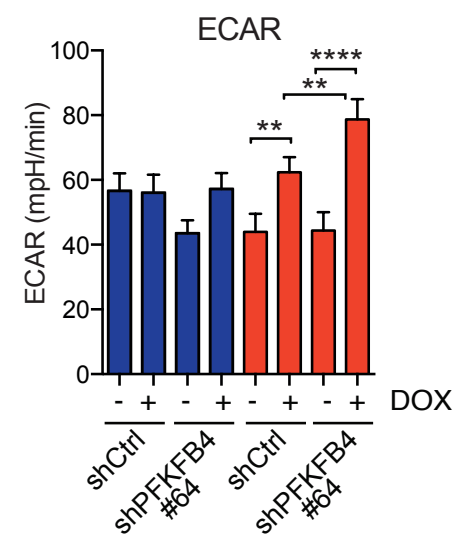

b

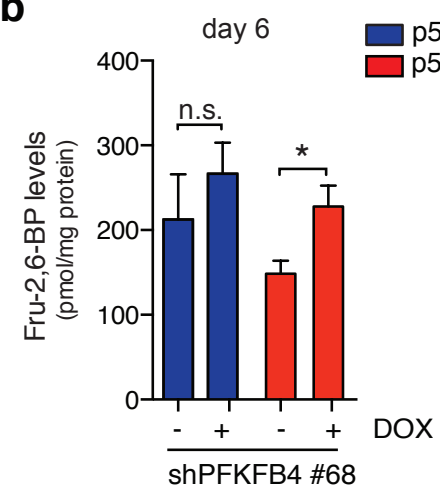

C

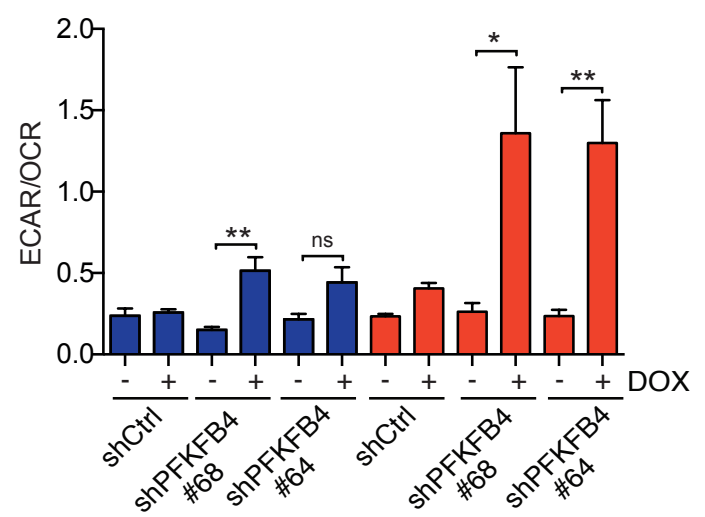

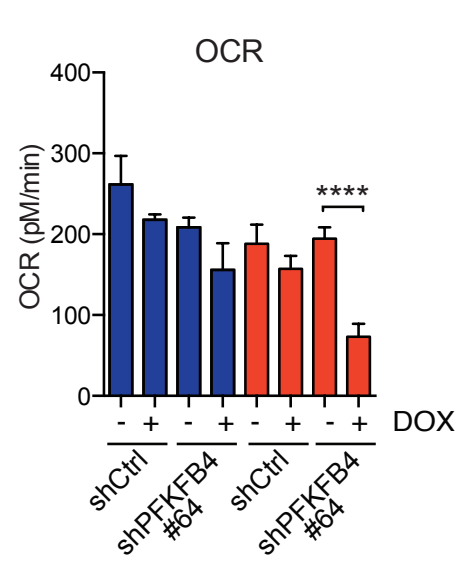

e

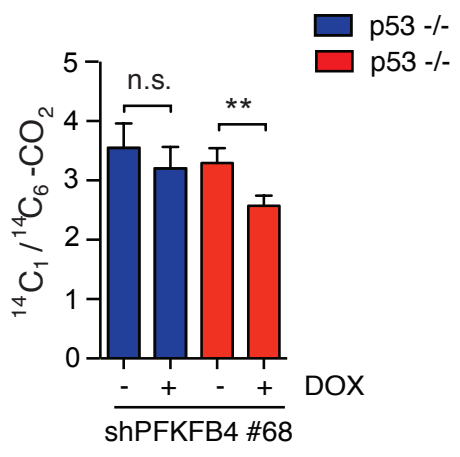



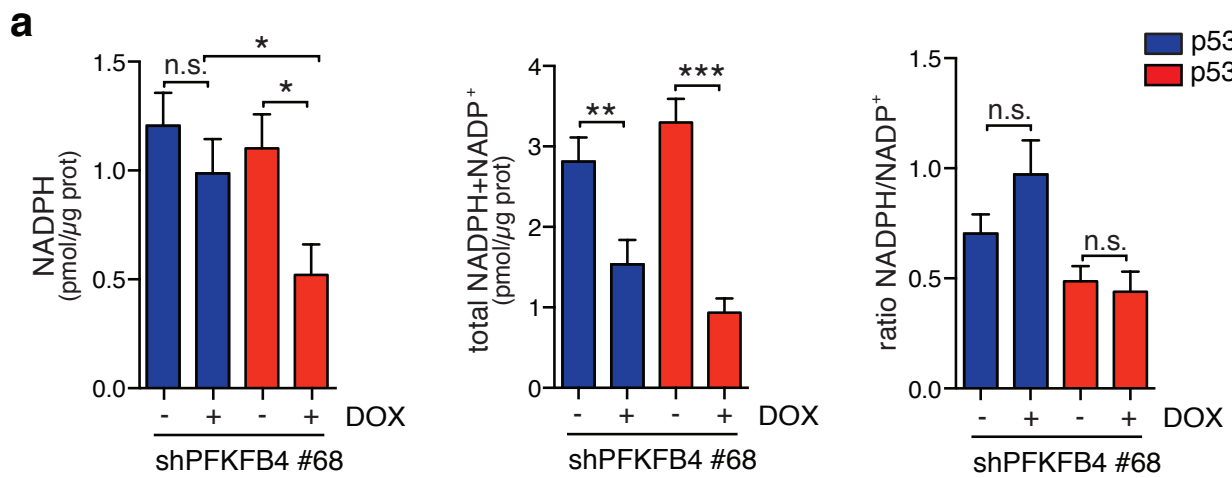

b

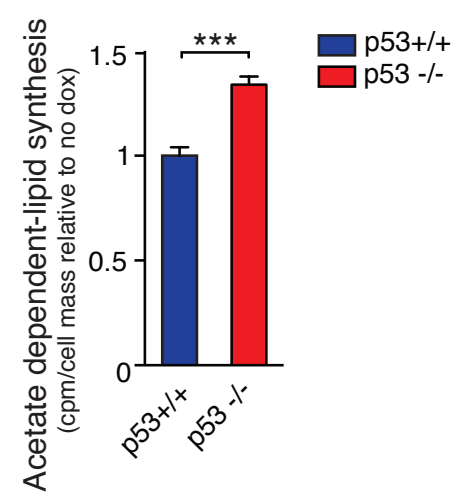

e

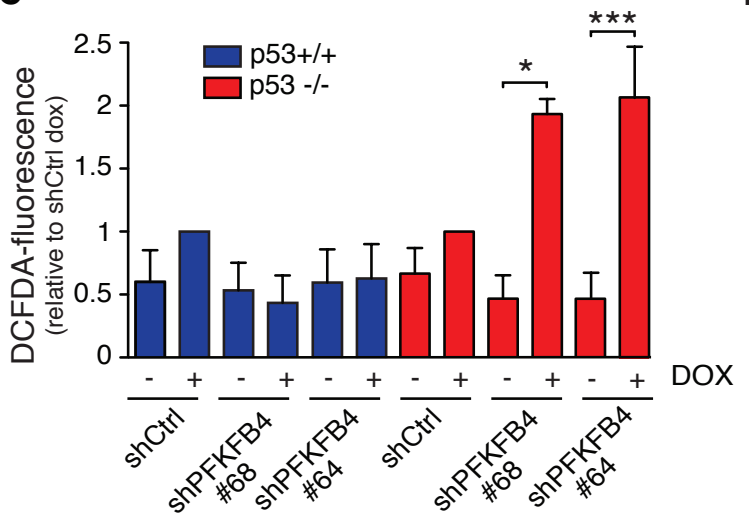

g
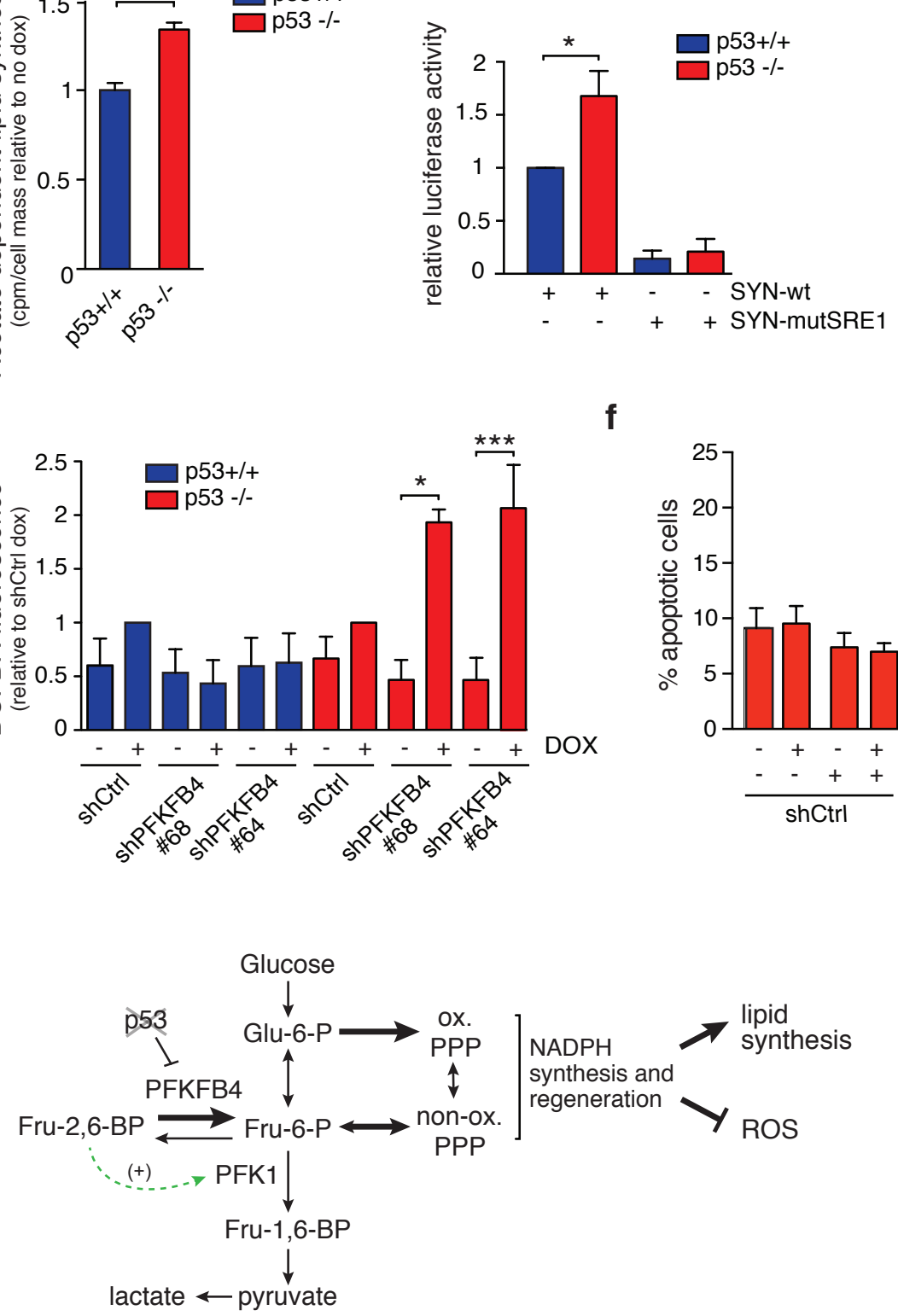

d
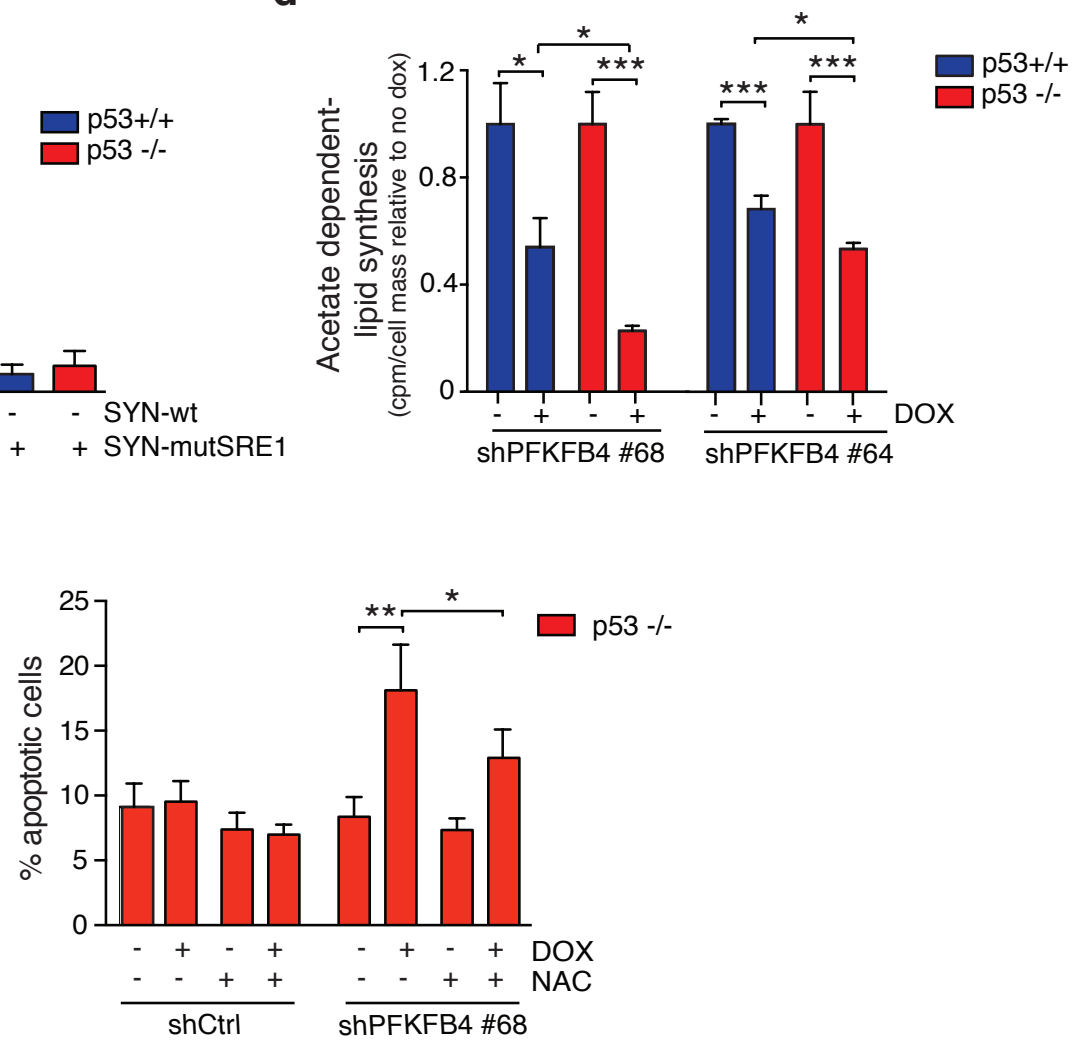
Ros et al. Figure 6
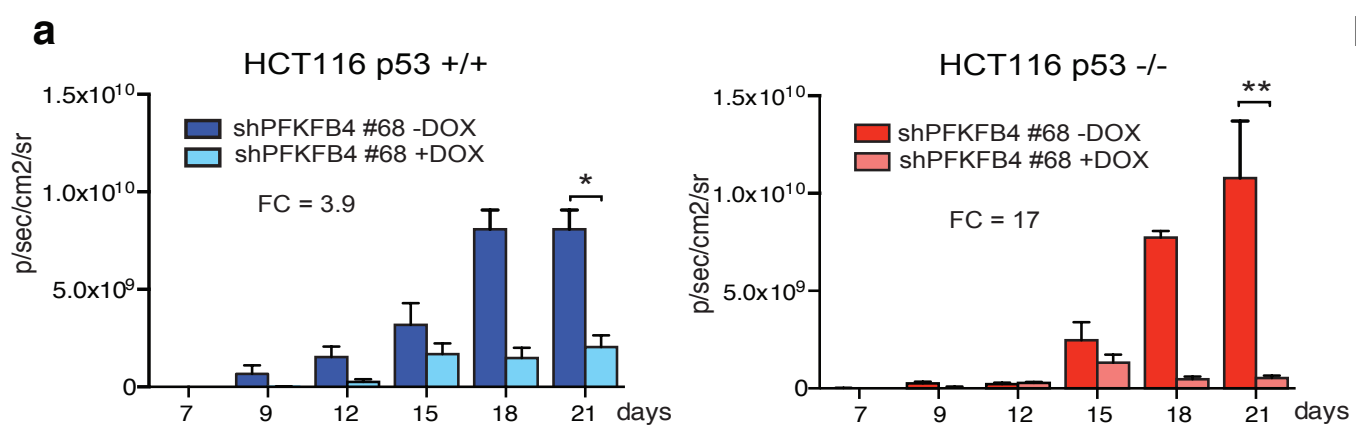

b

p53 +/+ p53-/-
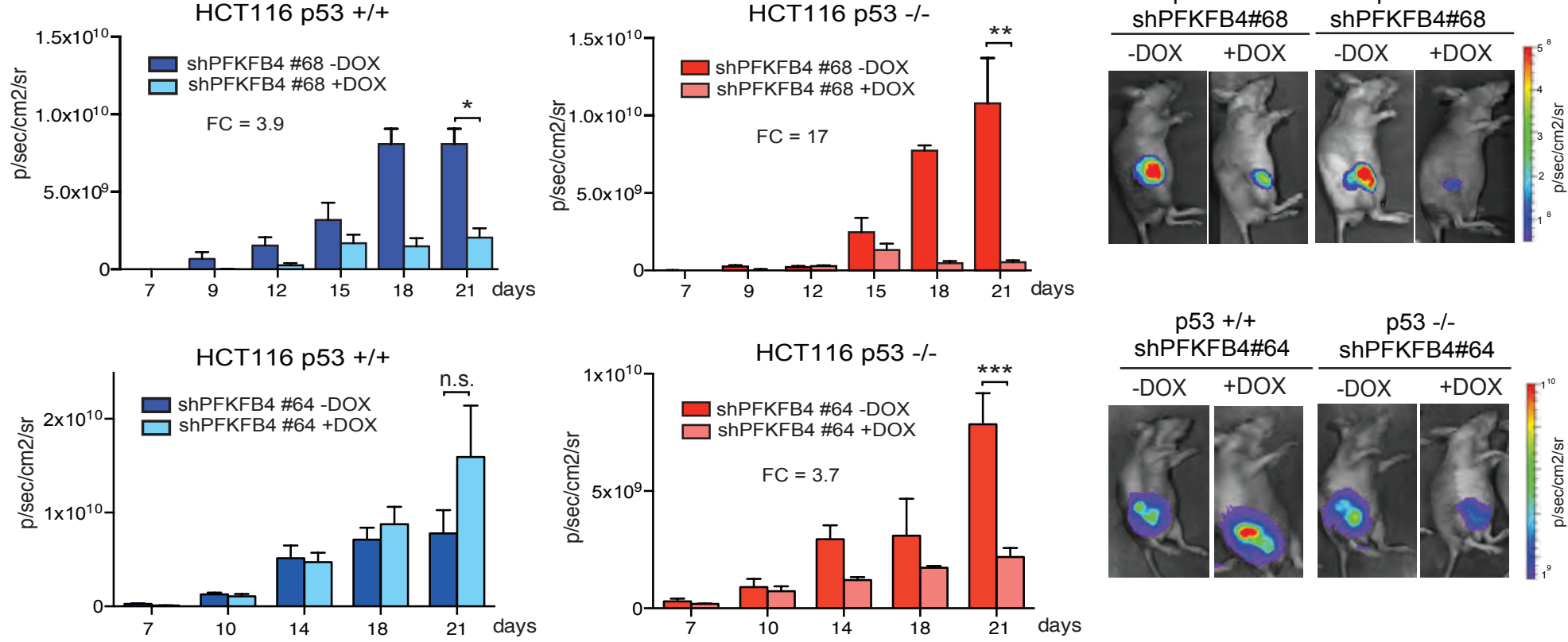

C
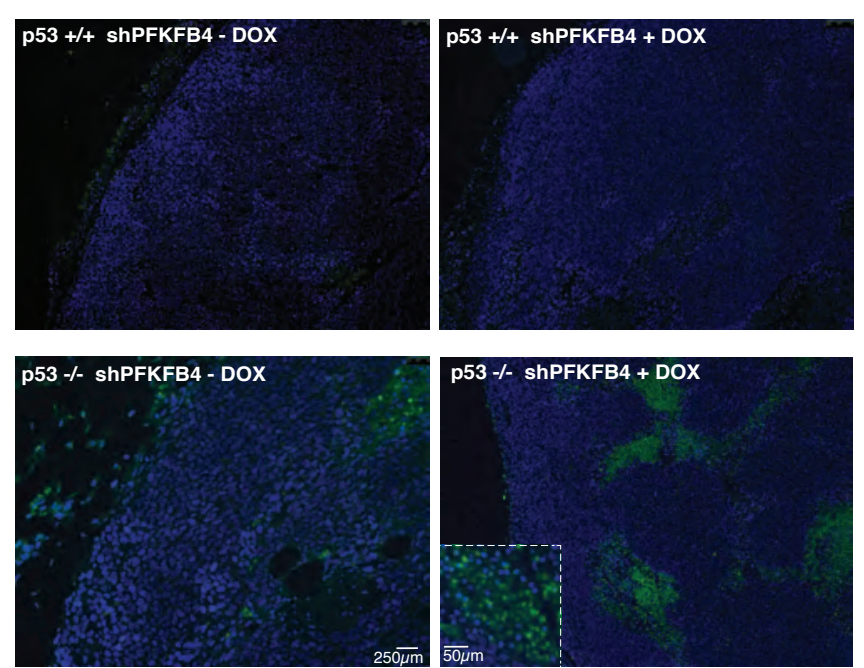

e

REACTOME FORMATION OF TERNARY_COMPLEX

(43 genes)
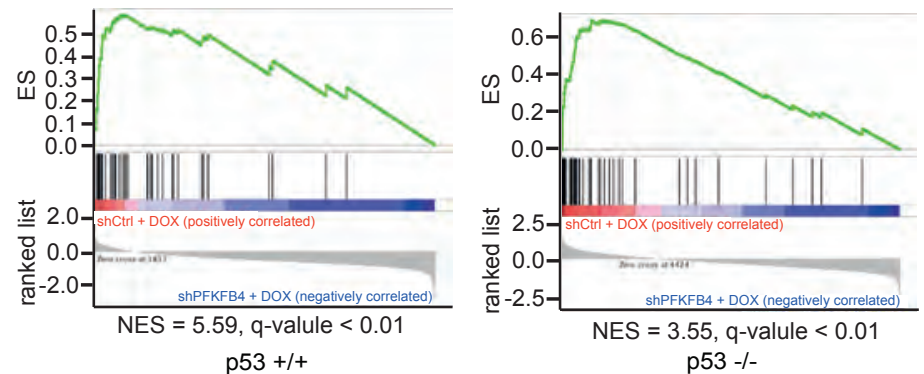

BILANGES SERUM AND RAPAMYCIN SENSITIVE GENES (60 genes)
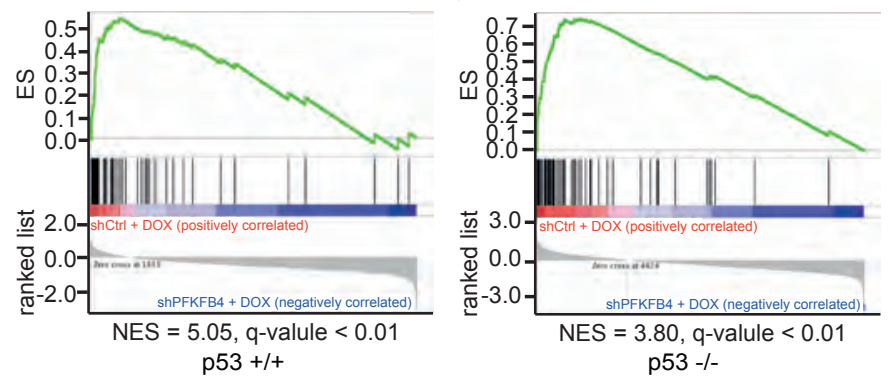

d
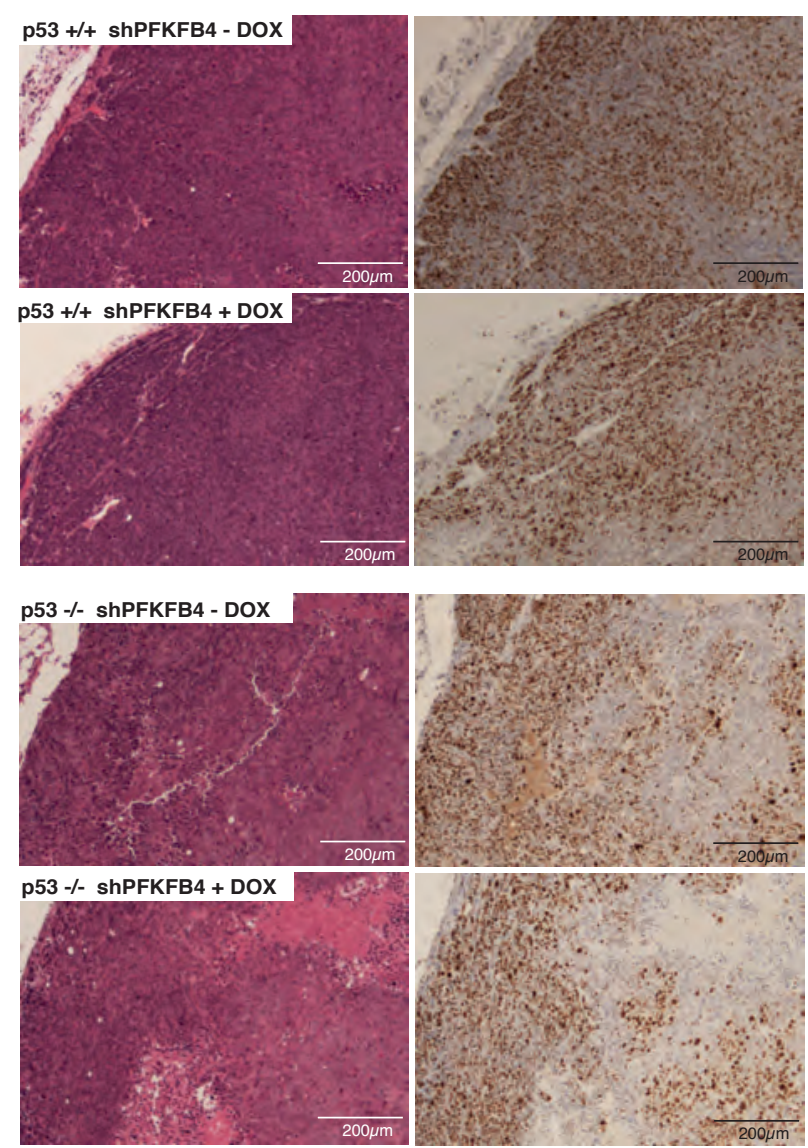